
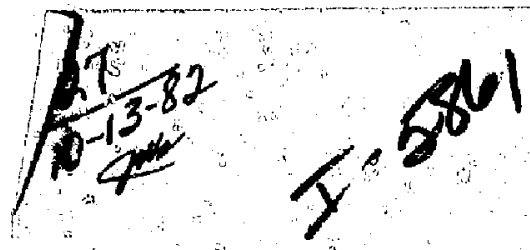

Dr. 903

UCID- 19562

A PHYSICS CONCEPTUAL DESIGN FOR THE

MFTF-B TRANSITION COIL

$\equiv$

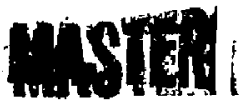

D. E. Baldwin

R. H. BuTmer

August 7, 1982

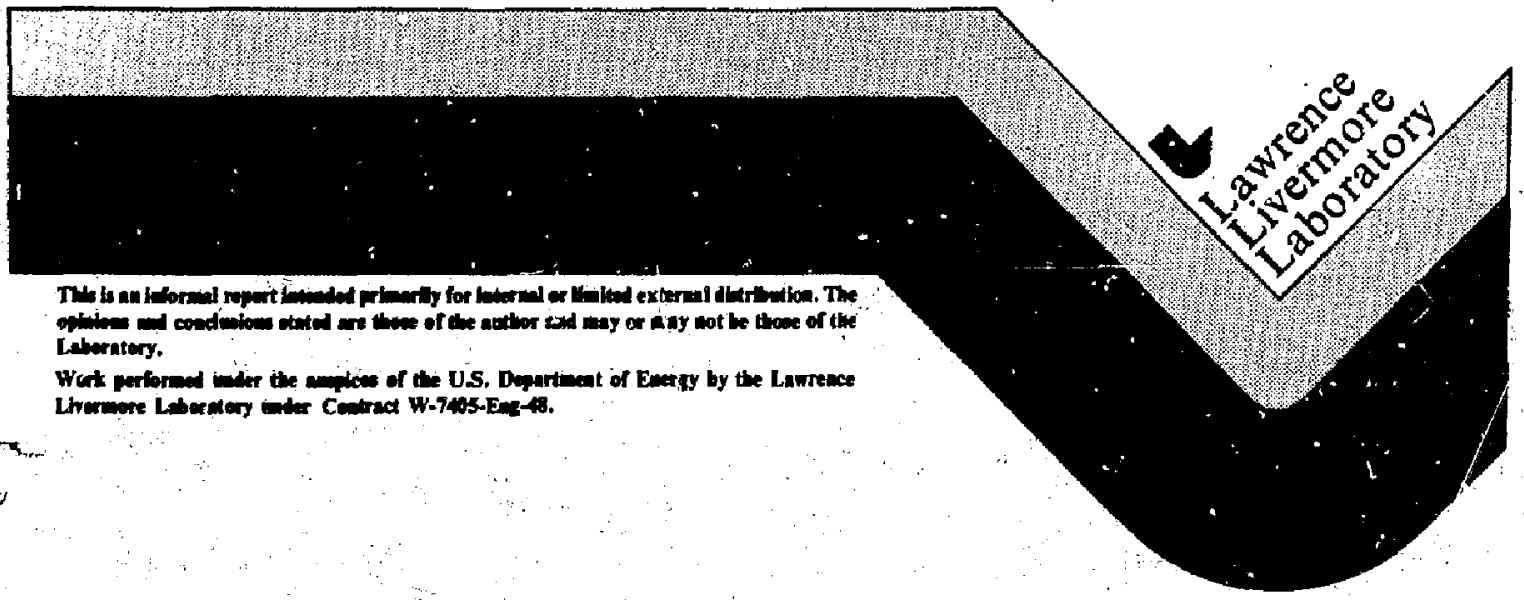

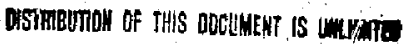




\section{A PHYSICS CONCEPTUAL DESIGN FOR THE MFTF-B TRANSITION COIL}

\section{INTRODUCTION}

At the April 1982 Review of the conversion of MFTF-B to an axicell configuration, the magnet design for the transition between the yin-yang anchor and the axicell did not simultaneously sati.ify impartant physics criteria. The nature and significance of this problem was identified by LLML, and the Review Panel requested to be informed of the success and nature of the solution whei the design had been completed. ' This document responds to that request. One end-region of the MFTF-B magnet set without transition coils is shown in Fig. Tz.

The physics aspect of the transition coil design has now been completed, with satisfaction of all of the criteria that had been identified at the time of the Review. The physics constraints related to finite- $\beta$ equilibria, $\beta$ limits due is curvature-driven MHD modes, and ion transport in thr central cell. These physics constraints had to be satisfied subject to certain nonphysics constraints. Principal among these were the geometric and structural features of the existing MFTF-B magnet set and the required access for neutral beams for pumping.

The body of this report discusses these constraints and their origins, Sec. II the physics and Sec. III the non-physics constraints. The Appendices discuss physics theory results that have been obtained since the cime of the Review and their implications for the design selected. Appendix A describes the limits on the radial electric field that are required for the validity of the transport criterion imposed on the transition magnet design. Appendix $B$ describes the calculation of curvature-driven $m=1$ ballooning modes that place a theoretical limit on central cell $B$ somewhat below that of pure interchange stability. In Appendix $C$, this axicell design for MFTF-B is compared $t$, a 

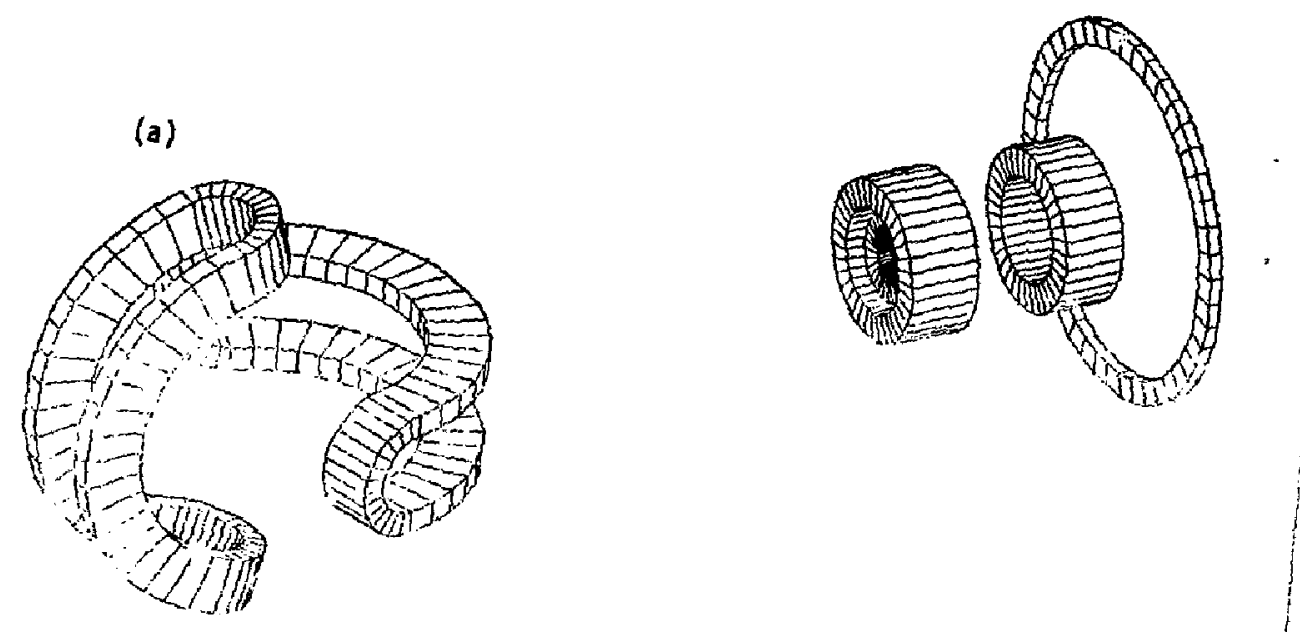

(b)

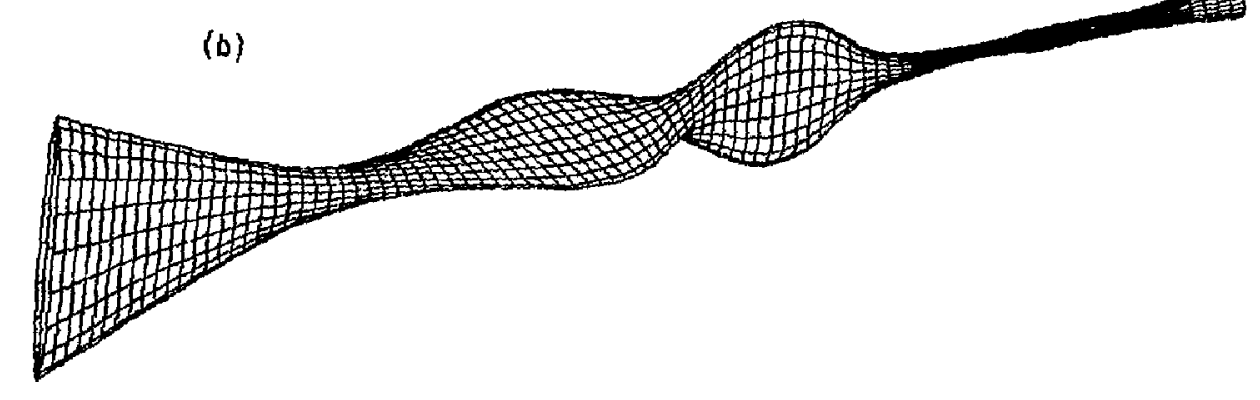

Fig. 1.

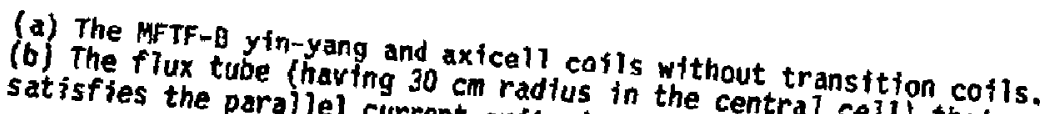
criteria. 
reactor-scale device in terms of dimensionless parameters that have been identified as important in determining the machine physics performance. In these variabies, the two quite different siza machines are found to be similar, usually to within a factor of two.

II. ORIGIN AND NATURE OF THE PHYSICS CONSTRAINTS

The equilibrium used in the MFTF-B is based on the work of Kaiser, Pearlstein, and Newcomb ${ }^{2}$ and has been incorporated into the code TEBASCO. The model is three-dimensional in the magnet field supplied by the magnetic design code EFFI. The equilibrium solution is based an an expansion to leading order in two parameters, the aspect ratio of radial to length scales and the plasma B-value.

A special class of pressure prafiles has been assumed throughout. If the magnetic field is written in terms of flux and angle-like coordinates,

$$
\underline{B}=\nabla \psi \times \nabla \theta
$$

elements of the pressure tensor are assumed to have the form

$$
P_{\perp, \|}=P_{L, \|}(B, \psi)
$$

such profiles have been termed "isorrophic". 2 They are compatible with drift equitibria when the plasma pressure is sufficient that $\nabla B-d r i f t s$ in the diamagnetically-depressed field dominate the curvature drifts.

When the magnetic: Tine curvature is expressed in covariant coordinates in the $\psi, \theta$ coordinate system 


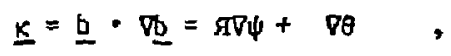

the geodesic curvature to causes the drifts of particles off of leve1 $\psi$ surfaces. This drift is responsible both for transport of ians across flux surfaces and for the generation of equilibrium current parallel to $B$.

\section{Transport}

Provided that the azimuthal drift frequency of ions in the transition is smal1 compared to their bounce frequency, so that to good approximation ions stay on a magnetic field line, their displacement in flux on one reflection from the end region is given by

$$
\Delta \psi=\frac{2 m c}{q} \int_{a x i c e) 1}^{\text {turnpoint }} d s\left(v_{11}+\frac{v_{1}^{2}}{2 v_{u l}}\right) b
$$

lons entering the transition region that suffer very large changes $\Delta \psi$ have orbits that are stochastic on their subsequent bounce motion through the solenoid. Even though with axicells only a small fraction of central cell ions reach the transition regions, the resulting stocthastic transport would constitute a prohibitive lass from the solenoid. This is the situation wich would prevail in MFTF-B without special attention to the magnetic design in the transition region to reduce $\Delta \psi$.

Ions that pass through the transition region have been accelerated into it by a potential that is several times their average central-cell energy. Therefore, $\Delta \psi$ is substantially independent of ion energy and pitch angle, other than that implicit in the turning point. Because passing ions are all reflected by the confining potential and thus have nearly the same turning 
points, their $\Delta \psi$ as given by $E q$. (1) can nearly be made to vanish by proper magnetic design. This design feature would nutl the line integral of the geodesic curvature up to the average turning point

$$
\int_{\text {axice } 11}^{\text {turnpoint }} d s=0
$$

In the long-thin approximation, the vacuum geodesic and normal curvatures can be written as

$$
\begin{aligned}
& 0=-2 \psi g(z) \sin 2 \theta \\
& g=h(z)+g(z) \cos 2 \theta,
\end{aligned}
$$

where $g$ and $h$ are functions of $z$ alone. Recircularizing the elliptical $f$ an on the inboard side of the yin-yang necessarily intraduces a large contribution to $g(z)$ and, therefore, regions of geodesic curvature of a given sign. Nulling an integral of the form Eq. (2) necessarily requires a region of opposite sign of 0 , obtained by fanning in the orthogonal direction. ${ }^{3}$ Therefore, transition regions satisfying Eq. (2) must have the character of two, successive orthogonal fans. A sample flux tube having this double-ellipse character is shown in Fig. 1b. In a transition region of fixed length, this dowble elifipticity will necessarily entail an increase in $g$ with the attendant increased drive for curvature-driven modes as a result of nulling the average geodesic curvature. As a practical matter, the magnitude of the geodesic curvature in the yin-yang is small (by $1 / 10$ or less) compared to that in the transition region, as shown in the sample 10 in Fig. 2. The integration in Eq. (2) is therefore insensitive to the turning point, and in practice integration can be stopped at the null in occurring near the inner yin-yang mirror. Figure 2 also illustrates the region in the transition of positive 10 , in addition to the 
$-6-$

B

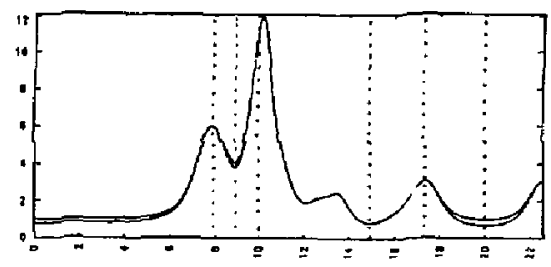

$z(m)$
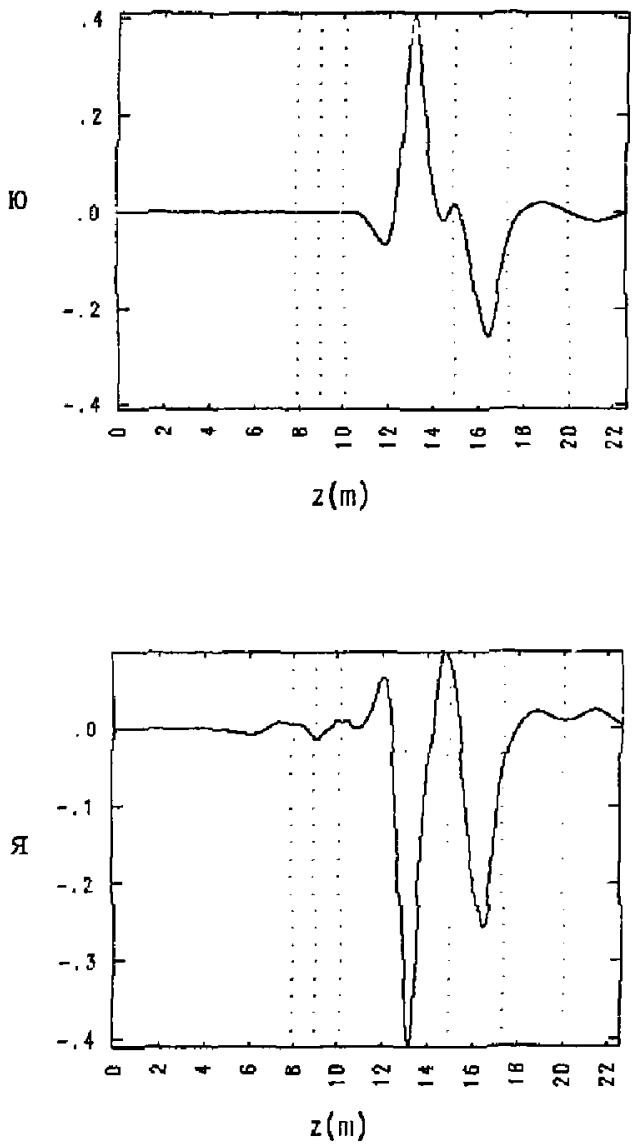

Fig. 2. Axial profiles of mod-8 and geodesic and normal curvature for one-half of MFTF-B. 
negative, that is characteristic of the double-ellipse nature of the flux tubes.

The increased magnitude of $g$ in the double-ellipse transition, in combination with the radial electric field, increases the azimuthal drift-pertransit. The nulling of Eq. (2) along a field line can become undone by an incomplete cancellation of positive and negative contributions of large magnitude, as seen by an ion along its actual drift orbit. The dominant drift is usually due to the electric field, and a drop in potential of $T_{e}$ in a plasma-radial scale length is usually enough to reintroduce stochasticity into the ion orbit. The minimum transport occurs when the eiectirc field is slightiy negative so that for typical passing ions there is a cancellation between the electric and curvature drifts. Details of this are discussed in Appendix A.

\section{Equilibrium Parallel Current}

The torodial Phrisch-Schulter current has an equivalent which occurs in a non-axisymetric open system. This current parallel to $B$ is given by

$$
B \frac{\partial}{\partial s}\left(j_{11 / B}\right)=-\nabla \cdot \stackrel{+}{\underline{j}}
$$

where the diamagnetic current has non-zero divergence. It must vanish where the pressure goes to zero at the ends of the device,

$$
j_{11}=B \int_{n=0}^{p_{1,11}=0} \frac{d s}{B} \frac{\partial}{\partial \psi}\left(p_{1}+p_{11}\right) n,
$$


and has a $\sin 2 \theta$ dopendence due to that of $\mathbf{x}$. It is important that $\mathbf{j}_{\mathbf{1}}$ vanish in the central cell. Otherwise, a long central cell would suffer large distortion.

In general, the requirement that $j_{\|}$vanish in the central cell leads to a required nulling of a different-weighted average of $\mathbf{b}$ than that of vanishing transport given by Eq. (2). However, well-pumped passing particle distributions have $P_{1, U} \propto B$ by flux conservation. For such distributions, the conditions become identical. Such distributions occur only in the transition, not in the yin-yang anchor. Thus, assuming separable pressure distributions, $p_{\perp, \|}(B, \psi)=$ $\omega(\psi) p_{\perp, 11}(B)$, a design which satisfies

$$
\int_{\text {transition }} d s x_{0} \quad \text { where } p_{1, \|} \propto 8
$$

and

$$
\int_{\text {anchor }} \frac{d s}{B}\left(p_{1}+p_{11}\right) 10=0 \quad \text { where } p_{2,11} \text { is mirror confined }
$$

will satisfy the zero-parallel current condition. It also almost nulls the integral in $\mathrm{Eq}_{\mathrm{G}}$. (2) because of the large magnitude of 10 in the transition compared to that in the anchor. The separation point of the decomposed integration can be taken at the zero of 10 near the inner anchor mirror, see Fig. 2. This general procedure is the one that has been followed in designing the MFTF-B transition region.

This dependence upon pressure profile represents an unsatisfactory aspect to the design procedure. The concern is whether parallel current might not be nulled in practice due to departures from the assumed $P_{1, H} \propto B$ nature of passing particle distributions, or whether parallel current might be significant during startup (even though $B$ would be lower). Two features mitigate this 
concern. (i) A design that has 50 antisymetric and B symmetric about the same point would by symmetry Jead to a vanishing of

$$
\int_{\text {transition }} \frac{d s}{B}\left(p_{1}+p_{11}\right) \text { D }
$$

for any $p_{1, u}(B)$. While this cannot be satisfied exact $7 y$, it does represent an ideal to be approached insofar as other constraints permit, and it relieves the sensitivity to the assumed pressure distributions. (ii) The strict nu1ling of $j_{11}$ is required only for very long central cells to avoid distortions proportional to the central cell length. In tandem mirrors of modest central cel] length the constraint is not so severe. In MFTF- $B$, we need only reduce the parallet current using a double-ellipse transition by a factor of five to ten below its value with a single-ellipse transition. This is sufficient to give equilibria with small central-cell distortions and acceptable transport rates.

The equilibrium solution determines the shape of the $\psi$-surfaces that are common to the various cells. When the central cell current has been nulled, these surfaces will still have a small distortion at the central-cell mid-plane due to the flux surface distortion generated in the end cells. They generally have also a small ellipticity in the yin-yang, due to the distortion of the yin-yang field by the transition coil.

\section{MHD Stability}

Curvature-driven stability in any tandem mirror relies heavily on FLR stabilization of higher $-m$ modes. In the extreme of this stabilization, only the m=l would exist, for which FLR is inoperative. Recently, Pearlstein, et a1., ${ }^{4}$ have developed a stability analys is for modes that are rigid 
displacements at each $z-v a l u e$, but can have differing displacements at different $z$-values. This is an $m=1$ ballooning for which the interchange is the flute limit. In general, this condition will give $\beta$-limits below the flute interchange condition. Because of its $m=1$ character, the solution must be carried outside the plasma, and wall-stabilization can be important provided the magnetic flux between the wall and the plasma is not large compared to that contained within the piasma.

This $m=1$ ballooning has replaced our previous flute interchange condition determining low-m (non-FLR-stabilized) modes. Details of the calculation are given in Appendix B. The principal drive occurs due to the pressure in the transition from those central ions that penetrate past the axicell to the plugging potential and their neutralizing electrons. The calculation is therefore dependent on the modeling which relates the ratios of these pressures we have used analytic axial-pressure profiles that mo'sl our fokker-Planck calculation for ion trapping and passing-partirle pressure distributions in the transition and anchor regions. As noted above, the double-ellipticity condition imposed to null the parallel current and the radial transport step-size also has the effect of increasing the narmal (as well as the geadesic) curvature in the transition region.

\section{II. MFTF-B STRUCTURAL CONSTRAINTS}

Beyond the physics considerations discussed in Section II, there are features of the MFTF-B configuration which impose special constraints on the magnet design. To some extent these features are due to the original singlemirror purpose of the MFTF magnet sets. They would not be present in a reactor magnet set design from the ground up for a tandem mirror application. 
Principal among these constraints is the heavy restraining structure on the outside of each yin-yang magnet element. This, in effect, extends the axiai length over which the fan-shaped character of the flux tube must be preserved roughly two meters beyond the peak of the mirnor into the trinsition region. ATthough one fan of the dauble-ellipse flux tube fits nicely within this structure, the major axis of the reverse ellipse is normal to the gap in the structure. This fan must therefore occur erivirely in the space between the outside of the structure and the axicell. Given that a fan having a certain integral of 10 must erist in this space, the length of the space therefore inversely affects the required magnitude of the curvatures in that region.

A second important constraint relates to access by the neutral beams that are required to pump and therefore maintain the passing-partic le distribution within the barrier. These must be injected at small angles to the local sagnetic fieid $\left(<20^{\circ}\right)$ while not dumping on magnets and structure near the plusmas. The favored access injects through the outer yin-yang lobe, misses the anchor ions, and intersects the flux tube at the inner mirror of the yinyang. The narrow aperture of the yin-yang at this point, in combination with the restraining structure, prohibit piacing transition coils within the jaws of the yin-yang and its structure.

$A$ less important feature of the MFTF-B magnet set is the identical nature of the yin and yang. While the currents in these two halves can be imbalanced in MFTF- $B$, there might be an advantage in reactor design to have slightly differing radii of curvature, sweep angles, etc., to accommodate the different field conditions seen on the outside and transition side of a given anchor cel1.

Finally, transition coils must be compatible with coil casings and i.angers, so as not to require extensive re-engineering of the yin-yang support structure. 


\section{THE TRANSITION-MAGNET DESIGN}

A prototype of the transition coil design which satisfies the constraints described in Secs. II and III is shown without supporting structure in Fig. 3 . It contains a principal (larger) transition coil that plays the same role as the sole transition coil in earlier designs in that it recircularizes the elliptical flux tube generated by the yin-yang. However, in this design this recircularization overshoots into a reversed ellipticity that in turn is recircuiarized by the secondary (smaller) transition coil.

In arriving at this dual transition-coil set, we have extended the length of each transition region to $7.25 \mathrm{~m}$ from the $6.0 \mathrm{~m}$ (measured from outer axicell to inner yin-yang mirror peaks) assumed at the time of the Review. This increased length permits generation of the required flux tube within the structural constraints and satisfaction of the parallel current conditicis, while not requiring too extreme local values of curvature. It also permits variaton around the point design. The increment of $1.25 \mathrm{~m}$ corresponds to one large-coil spacing in the central cell, so that this change implies only Elimination of two such coils from the central-cell and not a re-engineering of that region. Attempts to work within the $6 \mathrm{~m}$ spacing with the larger of the transition coils external to the yin-yang case resulted in somewhat lower $\beta$ values, considerably greater difficulty in nulling $j_{\| j}$, and larger curvatures. The sxtra $1.25 \mathrm{~m}$ is the best compromise between reduced center cell length and better machine performance. The impact of the reduced central cell length on the physics comparison between MFTF-B and the reactor is discussed in Appendix $C$.

The dual transition-coil set produces the required double-elliptical flux tube shown in Fig. 1b. The axial profiles of the mod- $B$ and geodesic and normal curvatures were those shown in Fig. 2 for the case that a null central cell 


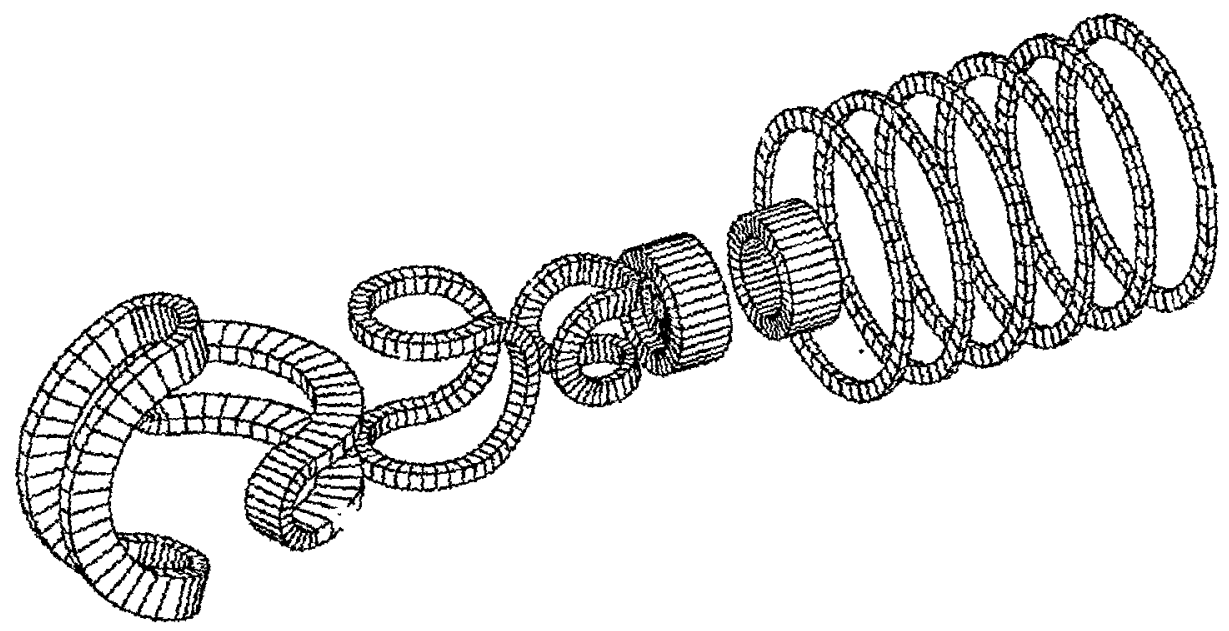

Fig. 3. The yin-yang, transition coils, and axicell of the MFIF-B magnet set. Only the conductor packs are shown. 
current has been achieved by nulling separately the contributions from the yin-yang and transition regions. The currents in the two coils required for this double nulling lead to a central-cell mid-plane fiux tube having the slightly distorted character shown in Fig. 4, characteristic of there being smal1 centrai-cell current. The residual distortion is chiefly generated in the and cells. This calculation used a profile $w(\psi)=(1-\psi)$. At the minimum of $B(z)$ in the anchor midplane, there is a residuai ellipticity of .9 due to imbalance of the yin-yang field caused by the transition coil. For this case, the large and small transition coils have currents of 3.6 and $2.7 \mathrm{MA}$, respectively.

The same coil set can be detuned away from a nulling of the central cell current by turning down the current in both coils and thereby approaching the single-transition-coil configuration, subject only to the smaller coil being sufficiently energized to keep the flux tube off of the coil casing. As an example, when the highly tuned configuration has been detuned by decreasing the small coil current by $25 \%$ and the large coil retuned to again null the yin-yang plasmla contribution to the current, the central-cell parallel current is not zero. However, for the central cell length in MFTF-B, the effect of this current is not too severe, and the ceritral-cell mid-plane flux tube has only the distortions shown in $\mathrm{Fig.} \mathrm{5.} \mathrm{(The} \mathrm{change} \mathrm{in} \mathrm{transport} \mathrm{is} \mathrm{equivalentiy}$ 5ma11, see Appendix B.) (Because of the reduced curvature, the $m=1$ ballooning limit in the central cell is raised to about $40 \%$. At this $\beta$ value, the flux surfaces have somewhat sharper corners; otherwise, they are similar.) Thus, a desirable operating point can be achieved by a fairly broad range of transition-coil operating currents, allowing for some ability to correct for imprecision in the calculational models. By such detuning of the transition coils, we also can ascertain the significance of the various physics criteria in the design and the impact of their non-satisfaction on machine performance. 

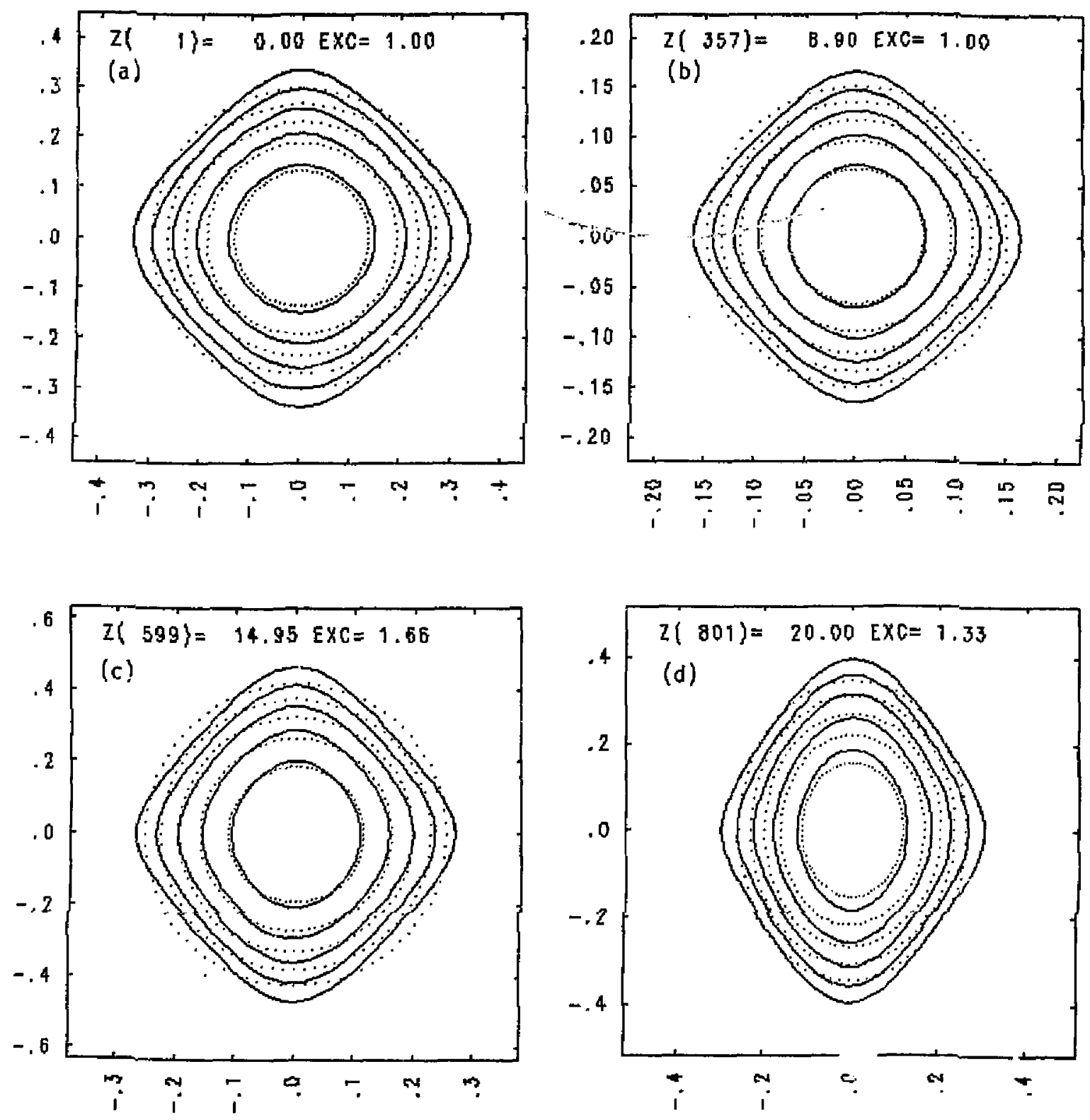

Fig. 4. Flux surface with nutled parallel current in the vacuum (dotted) and finite- $\beta$ fields (solid) at (a) Central cell mid-plane, (b) Axicell mid-plane, (c) Transition minimum-in-B, (d) Yin-yang minimum-in-B. For the finite- $B$ calculation: $B(y i n-y$ ang $)=0.55$, $B($ axice $])=0.1$ and $\beta$ (central cell) $=0.35$ with $\beta$ (transition) mapped from $B$ (central cel1). EXC $\equiv$ flux tube excentricity. 

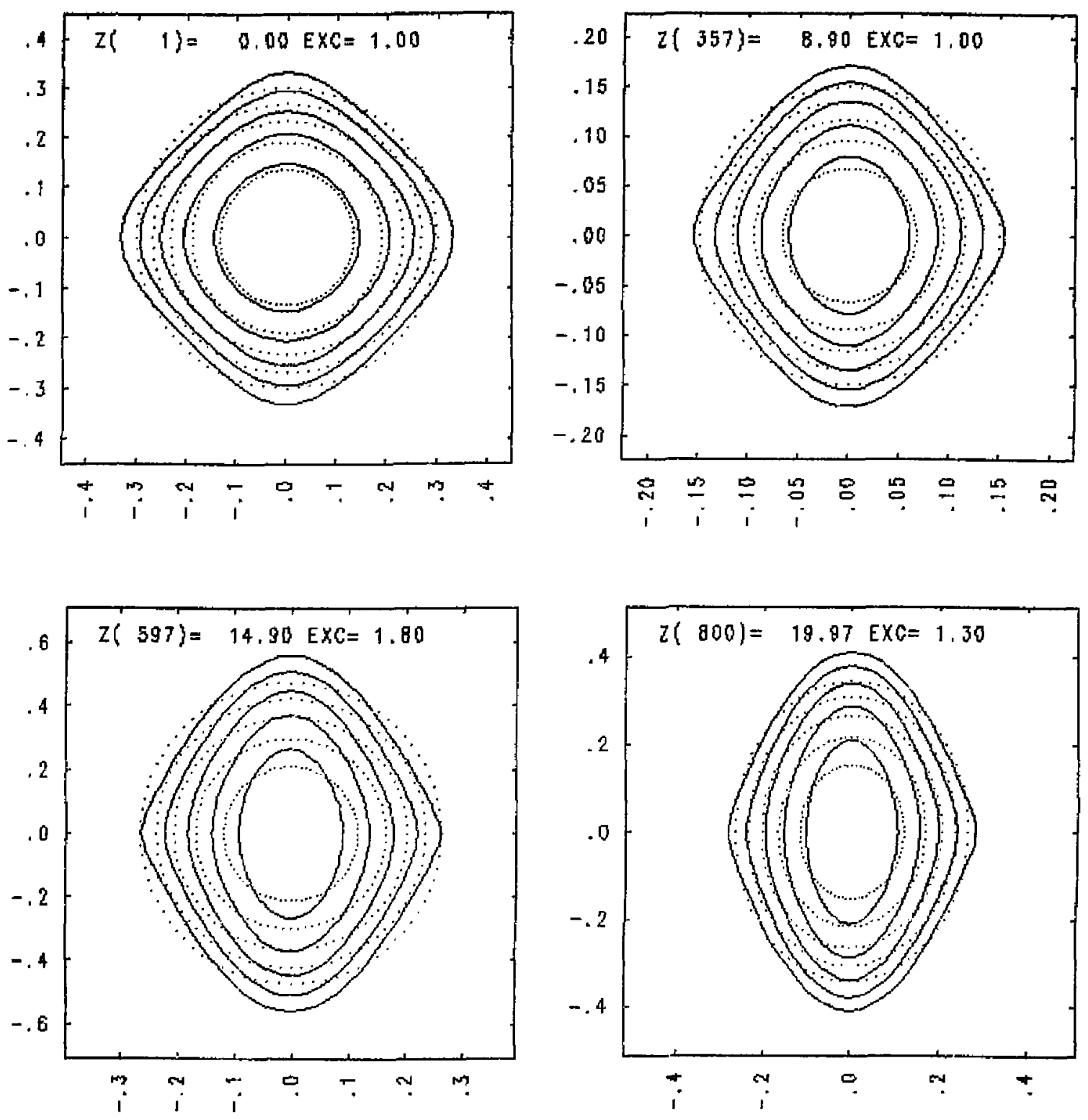

5ig. 5. Flux surfaces with incompletely nulled parallel curent in the vacuum (dotted) and finite- $B$ fields (solid) at (a) Central cell mid-plane, (b) Axice11 mid-plane, (c) Transition minimum-in-B, (d) Yin-yang minimum-in-B. For the finite- $B$ calculation: $B($ yin-yang $)=0.55$, $B$ (axicell) $=0.10$, and $\beta$ (central cell) $=0.35$ with $\beta$ (transition) mapped from $\beta$ (certral cel1). EXC $\equiv$ flux tube ellipticity. 
As discussed in Sec. II, radial transport due to ions' entering the transition region depends sensitively upon the radial electric field in the transition region. When the transition region has been pumped, the axis of the transition has a potential below the central cell and only about $2 \mathrm{~T}_{\mathrm{e}}$ above ground potential. The radial profjle will depend upon conditions such as the $T_{e}$ radial dependence, fueling as has been observed in TMX and PHAEDRUS, and on the radial effectiveness of the thermal barrier. We assume that we will be able to control the radial electric field as the earlier experiments have shown--at least over the ceniral core of the column. When the transitionregion radial electric field is assumed to vanish, we calculate a lifetime of 2 sec. for banana-regime transport in the double-núlied parallel current condition. This holds over an inner core of about $20 \mathrm{~cm}$ where the transport is non-stochastic. Qutside this core, the transport is again stochastic due to the larger central cell electric field near the piasma surface assumed in our model profile. The minimum transport occurs when the transition-region radial field is slightly negative so that it cancels the average ion curvature drift; the lifetime would be somewhat langer if this electric field could be maintained. More details concerning these transport calculations are included in Appendix $A$.

We assume that finite Larmor radius effects in the central cell are effective at stabilizing high azimuthal mode numbers. Assuming that all modes except $m=1$ have been stabilized by this means, several criteria can be used to calculate central cell B limits. For comparison, we hold fixed B(axiceli) $=0.10$ and $B$ (yin-yang) $=0.55$. These results are sumarized in Table 1 . The flute condition results from a pressure-weighted curvature average in the finite- $B$ fjelds; the original design goal had been 0.5 . The $m=1$ ballooning calculation due to Pearlstein, et al., ${ }^{4}$ summarized in Appendix B, depends on 
$-78-$

the assumed location of the flux-conserving wall. Table $l$ gives $\beta$ values for the wall at infinity and for two models for an elliptical wall which place the wall at minimum distances allowing for a $15 \mathrm{~cm}$ halo of cold plasma around the $30 \mathrm{~cm}$ hot plasma core.

\section{TABLE 1}

\begin{tabular}{|c|c|c|c|}
\hline & \multicolumn{3}{|c|}{$B_{c}($ on axis $)$} \\
\hline$j_{l l}($ central cel1) & Flute & $r_{w}=\infty$ & $\overbrace{15}^{n i n g} \mathrm{~cm} \mathrm{halo}$ \\
\hline Fully nuiled & .51 & .25 & $.31 \leftrightarrow .35$ \\
\hline $\begin{array}{l}\text { partially nulled } \\
\text { (75\% current in smalt coil) }\end{array}$ & .70 & .29 & $.35 \longleftrightarrow .40$ \\
\hline
\end{tabular}

Values are shown for the double-nulled parallel current condition described earlier and for the smali-coil current reduced by $25 \%$, yielding some parallel current. The improved "alues in the latter case result from the reduced magnitude of normal curvature in this partially relaxed case. The calculated ballooning degradation of central-cell $\mathrm{B}$ from the flute value measures the ability of the mode to isolate in reglons of bad curvature. The various wall models differ according to the mode energy expended in bending the field outside the plasma, allowing for the wall.

Finally, Fig. 6 shows the same magnet set in the presence of the two meters of structure on the outside of the yin-yang magnet. It illustrates the degree to which the structure restricted the design options in the transition region and dictated the physical size of the large transition coil. 


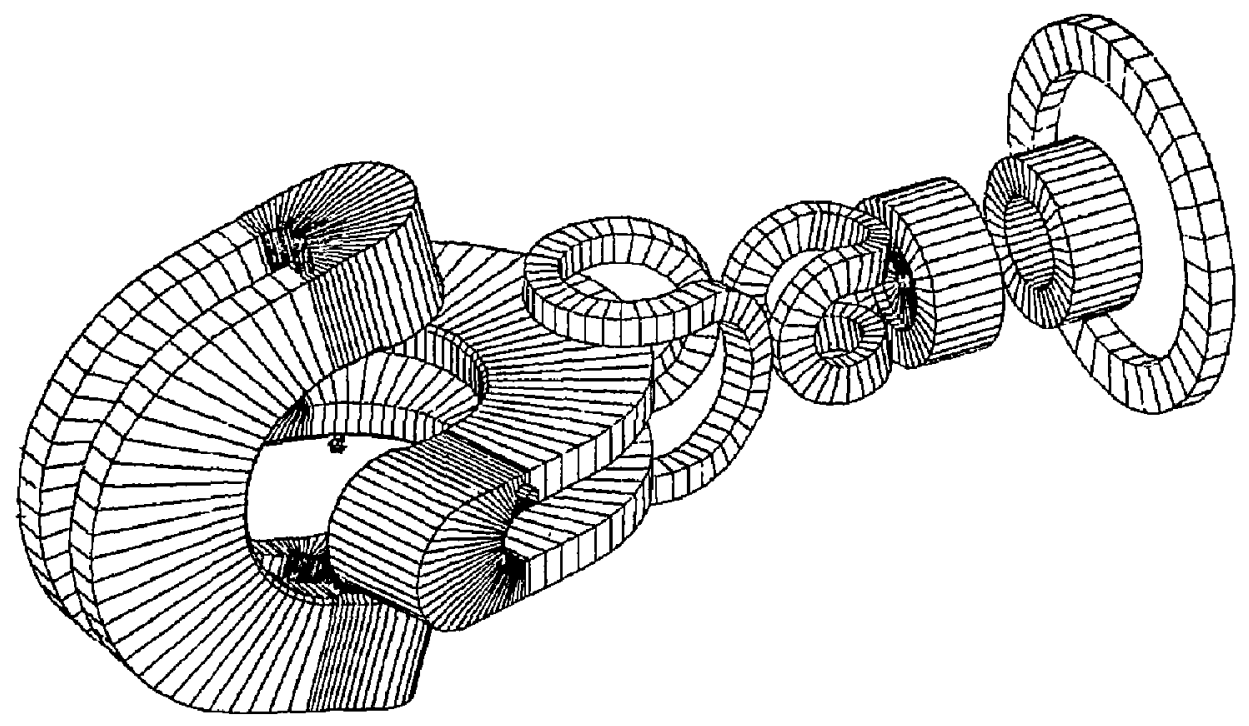

Fig. 6. The MFTF-B end-cell magnet set including transition coil and support structure on the yin-yang magnet. 
V. SUMMARY

The transition-magnet set described in the foregoing satisfies the physics design criteria set forth at the time of the Review. Principally, these criteria require that the Iine integral of the geodesic curvature in the transition region vanish with zero central-cell parallel current, and that the flute-stability $\beta$ limit exceed 0.5. By adopting a dual transition-coil set and extending the transition region to $7.25 \mathrm{~m}$, ihe design has sufficient flexibility to test the significance of these criteria in their original form and as they have been modified by refinements in the theory.

\section{ACKNOWLEDGEMENTS}

We wish to thank T. K. Fowler and B. G. Logan for numerous helpful discussions in the course of evolving this design and the several members of the theory group for carrying out supporting calculations. 
$-21-$

APPENDIX A: Transport 


\section{APPENDIX A: Transport}

(Based on wark by R. H. Cohen and J. A. Byers)

Drift transport in a tandem mirror has its origins in the changes in flux coordinate $\Delta \psi$ experienced by a particle on reflecting from a non-axisymetric end cell. If on an axial transit through the solenoid the particle suffers an angular displacement $\Delta \theta$, due chiefly to the radial electric field in the solenoid, the associated motion is non-stochastic provided

$$
\Delta \psi \frac{d \Delta \theta}{d \psi}<\frac{1}{2}
$$

Stochasticity can therefore be introduced either by large $\Delta \psi$ or by large shear in the central cell radial electric fjeld. When the motion is non-stochastic, particle collisions in the presence of the drift motion give rise to various forms of neoclassical and resonant transport. In the axicell geometry, these latter transport rates are manageable. The imps.ant point is to avoid stochasticity.

On one reflection from an end region, the change in flux is given by

$$
\Delta \psi=-\frac{2 m c}{q} \int_{0}^{s_{t}} \frac{d s}{v_{11}}\left(v_{11}^{2}+\frac{v_{1}^{2}}{2}\right)
$$

where $s$ measures distance along an actual particle drift orbit, and $s_{t}$ is the particle turning point. At the same time there is a change in angle variable given by

$$
s \theta=2 \int_{0}^{s t} \frac{d s}{v_{11}}\left[\frac{m c}{q} g\left(v_{11}^{2}+\frac{v_{1}^{2}}{2}\right)+c \frac{\partial \phi}{\partial \downarrow}\right]
$$


When $\delta \theta$ is sufficiently small, the particle stays on the field line during reflection. When in addition $v_{11} \gg v_{1}$ and $v_{\|}=$constant, as it is with acceleration into the transition region, $\Delta \psi$ becomes small with

$$
\int_{0}^{z} d z b
$$

giving the design condition described in the text.

Increasing the mirror ratio of, the MFTF-B yin-yang from two to three has the effect of increasing 101 in the transition region. Without attention paid to reducing or nulling $\int \mathrm{bodz}$, the increase in $\Delta \psi$ of those ians entering the transition would result in their stochastic transport unless the central-cell racial field were quite small. The double-ellipse transition coil design described in the test can null $\int$ dzlo. However, because

$$
\int_{0}^{z_{t}} d z 10 \ll \int_{0}^{z^{t}} d z 1001
$$

there is the possibility that a nulling of $\int d z l 0$ can be undone by a relatively small $\delta \theta$.

We calculate transport lifetimes for this more complicated situation by two complementary approaches. The first employs an orbit code developed by J. A. Byers that uses actual EFFI-generated magnetic fields and model analytic potentials in flux variables. A statistical sampling is sufficient to determine the conditions for stochasticity. In the second approach, R. H. Cohen expands Eqs. (AI) and (A2) for small $\delta \theta$ and obtains a two step map for the arbit variables in the central cell. This map agrees well with the Byers code results, and it permits determination of stochasticity conditions from the 
magnitudes of certain integrals of the curvatures, products of curvatures, and the various electric field profiles. While more quantitative work is still in progress, we can both draw general conclusions and obtain specific numerical results from these two approaches:

- A nonzero radiai electric field in the center cell and transition regjon is necessary for stochastic transport to occur. That is, the transport is nonstochastic if $E_{r}=0$ everywhere. But this is unlikely to occur.

- For the design parameters with a potential drop of $50 \mathrm{kv}$ in the center cell and $20 \mathrm{kv}\left(\approx \mathrm{T}_{\mathrm{e}}\right)$ in the transition region, the transport of passing center cell ions is stochastic over most of the plasma radius.

- A null electric field in the transition region, obtained sy biasing the entire axial profile of $\phi$ by a $-20 \mathrm{kv}$, leads to a valje of $\Delta \psi$ that gives nonstochastic transport for passing center cell ions out to about $20 \mathrm{cmi}$ (measured in the center cell). Based on resonant-banana transport, passing ions below this radius have a transport time of about $2 \mathrm{sec}$. Beyond this radius the stochasticity condition is violated for passing ions. This is based on a model central cell potential profile that vanishes at the plasma surface $(r=35 \mathrm{~cm})$. The nonstochastic volume would increase if the potential were not forced to zero at the plasma surface. We show an example of a stochastic orbit compared to a nonstochastic orbit in Fig. A1. 
- A positive electric field abets the normal-curvature drifts for ions. Therefore, a negative electric field in the transition that cancels the $\theta$-drift yields the least transport, for then the condition

$$
\Delta \psi \propto \int \operatorname{odz}
$$

pertains (for an average ion), and this can be designed to zero.

- A negative electric fielf in the transition region, consistent with a negative potentia) in the range of -8 to $-15 \mathrm{kV}$ (again vanisining at the plasma surface), increases the stochasticity radial boundary to somewhere in the range $25-30 \mathrm{~cm}, i . e$. most of the plasma volume is now nonstochastic.

- These conclusions about the benefits of control of the radial electric field are not peculiar to the particular coil set selected but rather common to all those that have been examined. 


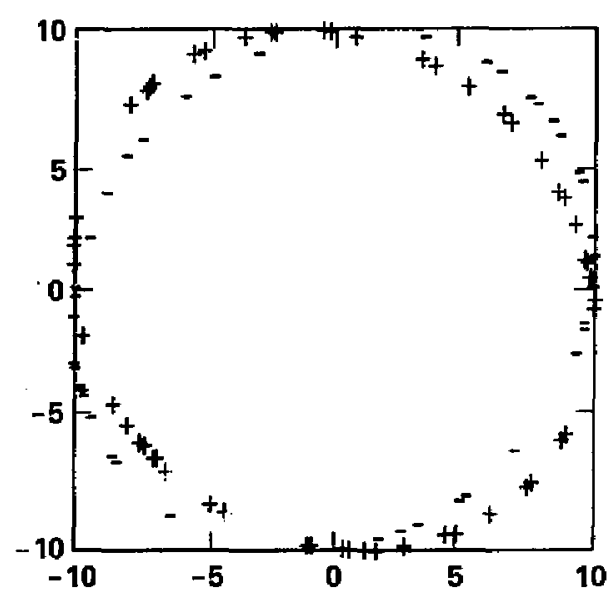

(a)

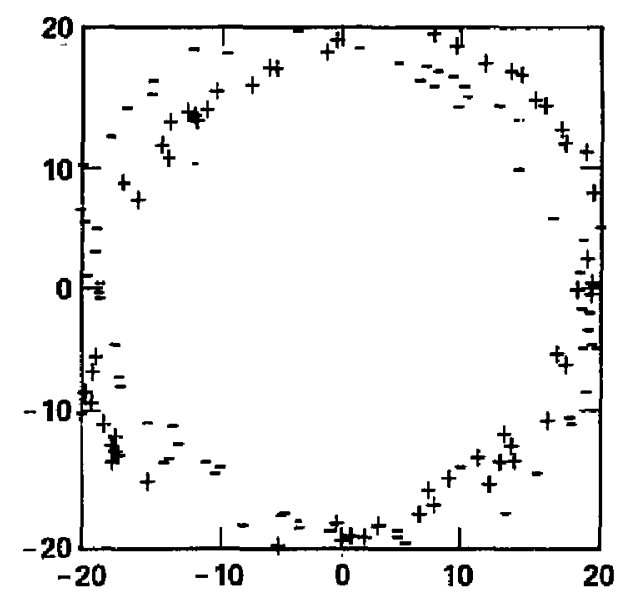

(b)

Fig. Al. Comparison of a nonstochastic orbit (a) to a stochastic orbit. (b) Particle positions in the center cell midplane are indicated with a "+" or "-" depending on direction of axial travel. The nonstochastic orbit with initial radius at $70 \mathrm{~cm}$ shows a clean elliptical curve whereas the stochastic orbit with initial radius at $20 \mathrm{~cm}$ shows breakup of the elliptical shape. This particle in fact escapes to plasma surface $(r=35 \mathrm{~cm})$ if observed over a longer timescale. 
$-27=$

APPENDIX B: $m=1$ Ballooning Stability 


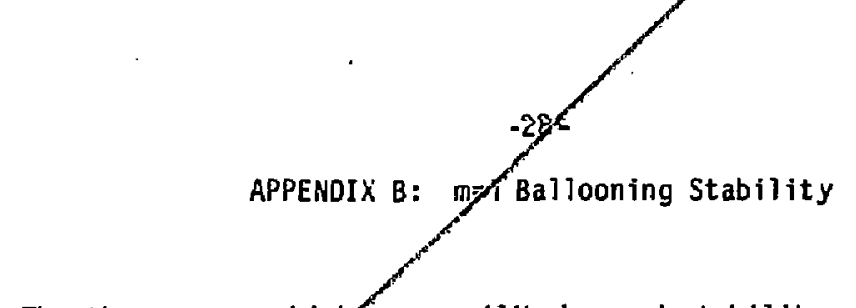

The theory upon whick our equilibrium and stability calculations are based is described in the IAEA preprint reproduced at the end of this Appendix. The $m=1$ ballooningestability theory is described in Sec. 3.2 of that report. The influence, ff wall stabilization was illustrated for the TMX-U magnet set using a wodel confocal elliptical wall that contains an amount of magnet flux betwen the wall and plasmas equal to that within the plasma.

Figure $B I$ shows results of similiar calculations by T. B. Kaiser for the MFIF-B magnet set with transition coils described in the body of this report. For these calculations the finite-wall mode 1 placed the wall a constant distance from the central cell circular flux tube and the long side of the elliptical fans given by $\left(r_{\text {wall }}-r_{c}\right)$ where $r_{c}$ is the central cell radius; near the tips of the ellipses it was less. This model thus represents an upper bound on the stabilizing influence of the wall whereas the $r=\infty$ case represents a lower bound. 
(a)
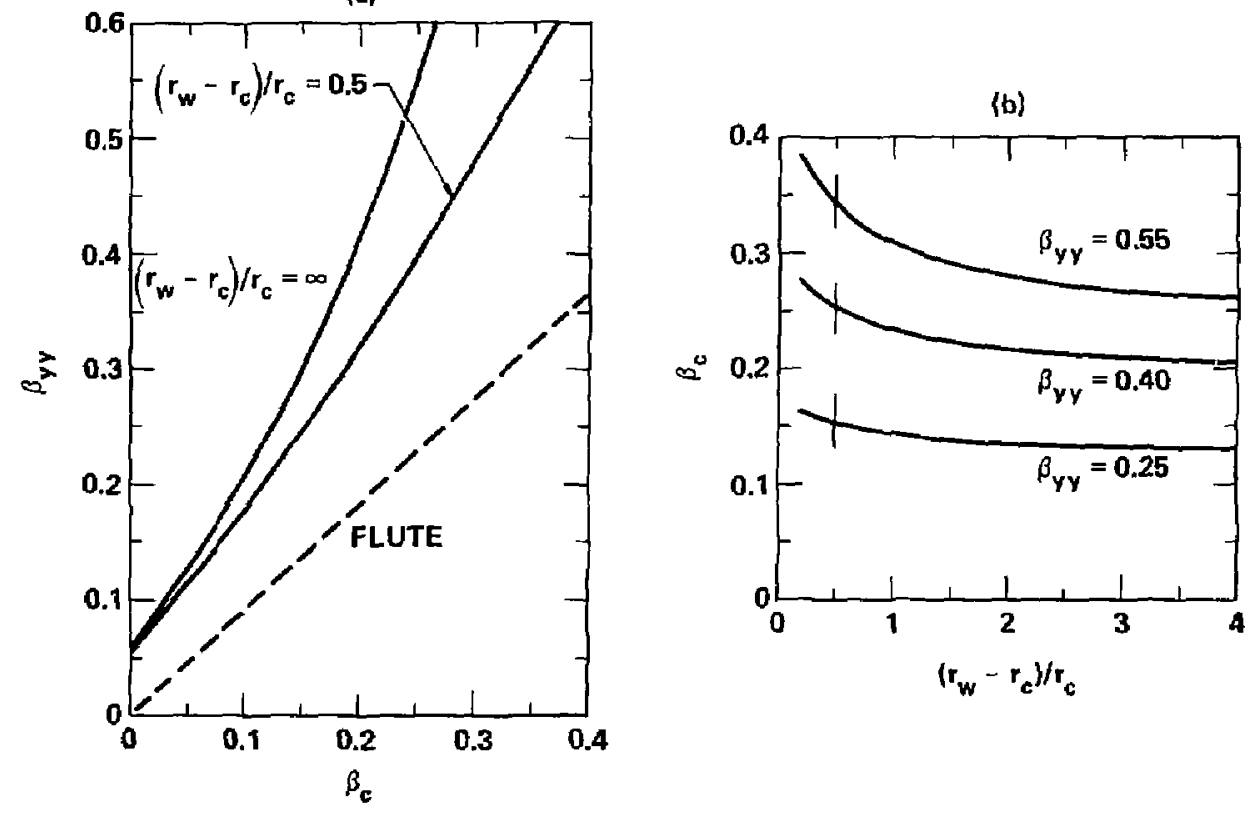

Fig. B1. Stability boundaries for the $m=1$ ballooning mode (a) $B$ (yin-yang) vs $\beta$ (central cell) for the two values of $\left(r_{w}-r_{c}\right) / r_{C}$, together with the flute interchange limit. Note that due to pressure in the axicell, the $\beta_{c}=0$ intercepts do not asymptote to the flute limit, which assumed no such pressure. (b) B(central cell) vs $\left(r_{w}-r_{c}\right) / r_{C}$ for three $B(y i n-y a n g)$ values. The tick marks in (b) indicate the $r_{w}=45 \mathrm{~cm}, r_{c}=30 \mathrm{~cm}$ value $c$ ited in the text. 


\section{GYROKINETIC EQUILIBRIUM AND STABILITY}

R.H. Buimer, T.B. Kaiser, H.M. Nevins W.A. Newcomb and L.D. Pearlstein H.R.Strauss and S. Wollman* M. Wakatani ${ }^{+}$

This paper was prepared for submittal to 9th International Conference on Plasma Physics and Controlied Nuclear Fusion Research, Baltimore, Maryland, 1-8 September 1982

August 2, 1982

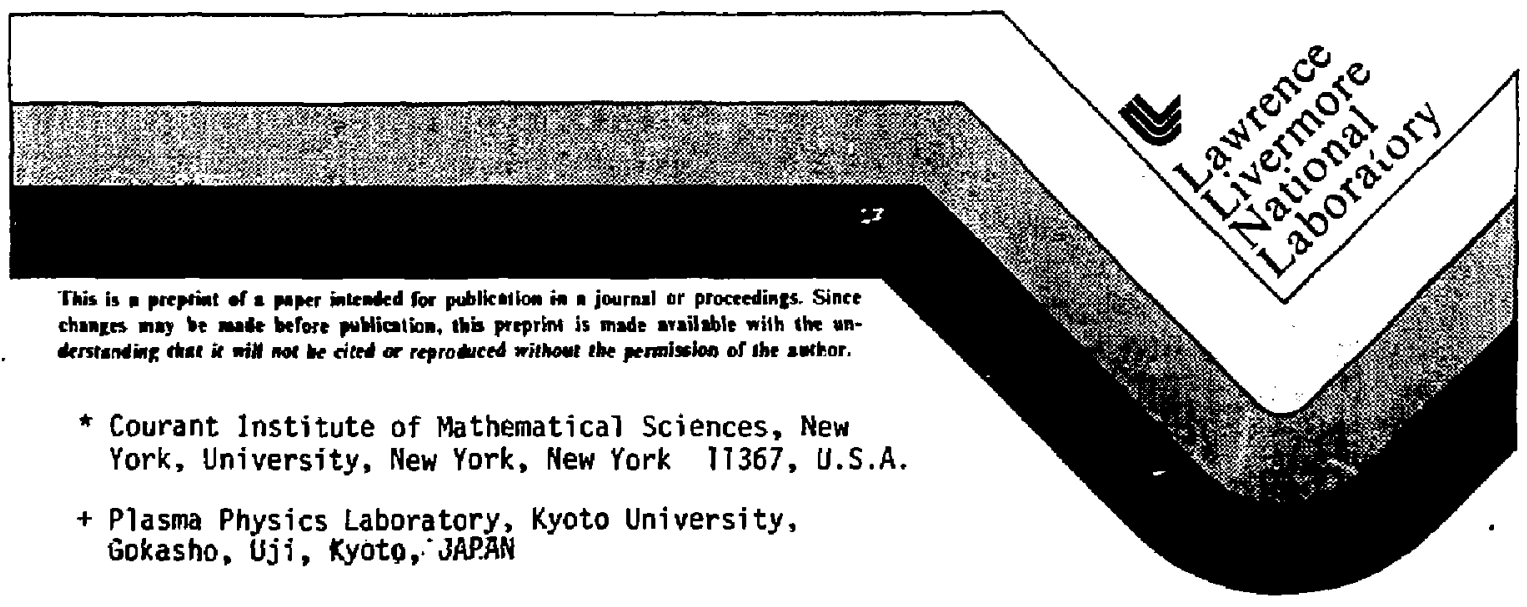



AND CONTROLLED NUCLEAR FUSION RESEARCH

Baltimore, USA, 1-8 September 1982

GYROKINETIC EQUILIBRIUM AND STABILITY IN QUADRUPOLE

TANDEM MIRRORS

R. H. BULMER, T. B. KAISER, W. M. NEVINS, H. A. NEWCOMB and

L. D. PEARLSTEIN

Lawrence Livermore National Laboratory, University of

California, Livermore, California 94550, U.S.A.

H. R. STRAUSS and S. WOLLMAN

Courant Institute of Mathematical Sciences, New York

University, New York, New York 11367, U.S.A.

M. WAKATANI

Plasma Physics Laboratory, Kyoto University, Gokasho, Uji, Kyoto, JAPAN

This is a preprint of a paper intended for presentetion at a scientific meting. Becausa of the provisionat natura of its content and since changes of substance or detail may have to be mode before publication, the proprint is made available on the understanding that it will not be cited in the literature or in any way be reproduced in its present form. The views exprused and the stetements made remain the respontibility of the named author(s); the viam do not necessurily raflect those of the govemment of the designating Mamber Stste(s) or of the designating organization(s). In aurticular, neither the lAEA nor any other orgunization of body sponsosing this meating can be hald responsiore for any meterial ruproducad in stis pruprine. 
IAEA-CN-4 I/G-2

\section{GYROKINETIC EQUILIBRIUM AND STABILITY IN QUADRUPOLE TANOEM MIRRORS}

\section{ABSTRACT}

This paper discusses recent theoretical work on the equilibrium and stability of quadrupole tandem mirrors in the paraxial limit. It reviews calculations of three-dimensional equilibria by means of a $\beta$-expansion technique which lead to an understanding of the important role played by parallel currents and the corollary importance of careful ciesign of the structure of the vacuum geodes ic curvature. The previously predicted [3] scaling with central-cell length of the finite- $B$ distortion of vacuum flux surfaces is shown to saturate because of finite orbit effects. An adaptation to tandem geometries of the reduced MHO technique for calculating high-B threedimensional equilibria is described. This approach uses the paraxial expansion to resolve the time-dependent relaxation to equilibrium inco three distinct timescales on which the motion can be followed independently. Regarding stability, it is shown that kinetic effects suppress ballooning modes of shortto-moderate perpendicular wavelength; in the limit that such effects are dominant only rigid modes are possible. The stability of the latter modes is investigated within the context of the energy principle. Results of equilibrium and stability calculations for the TMX-U and METF- $B$ experiments at Livermore are presented.

\section{INTRODUCTION}

- Present tandem mirror configurations consist of an axisymetric central cell, which serves as the principal confinement region, flanked by quadrupole end cells, which provide magnetohydrodynamic (MHD) stabilization as well as electrostatic plugging. The quadrupole anchors render the guiding-center equilibrium and stability problems threedimensional. Fortunately, however, tandem mirrors are large-aspect-ratio devices: magnetic field components and scale lengths parallel to the magnetic axis are large compared to those perpendicular to the axis. This ordering allows an important simplification of the equilibrium and stability problems. This simplification--commonly referred to as the paraxial or long-thin approximation--is achieved by expanding the equations that determine guiding-center equilibrium and stabi]ity in powers of the inverse aspect ratio, $\lambda[1]$. We report here equilibrium and stability calculations for tandem mirrors carried out within the context of the paraxial expansion, and their application to the magnet design 
of the two tandem configurations at Lawrence Livermore National Laboratory--the Tandem Mirror Experiment, (TMX-U), and the Mirror Fusion Test Facility (MFTF-B) which is now under construction.

\section{EQUILIBRIUM}

\subsection{Zero-Gyroradius, Low-B Limit}

We first discuss equilibrium. If terms of relative $0\left(\lambda^{2}\right)$ are neglected, the relevant equations reduce to [2].

$$
\begin{aligned}
& p_{\perp}+\frac{1}{2} B^{2}=\frac{T}{2} B_{v}^{2}(z) \\
& p_{1}=-B^{2} \frac{\partial}{\partial B}\left(\frac{p_{1}}{B}\right) \\
& \frac{\partial(x, y)}{\partial(\psi, \theta)}=\frac{1}{B} \\
& v \frac{\partial Q}{\partial \psi}=-B\left(\frac{Q}{B^{2}} i\right)^{\prime} \\
& i=\frac{1}{B}\left[\frac{\partial\left(x^{\prime}, x\right)}{\partial(\psi, \theta)}+\frac{\partial\left(y^{\prime}, y\right)}{\partial(\psi, \theta)}\right]
\end{aligned}
$$

Here we have used a flux coordinate system $(\psi, \theta, z)$ in terms of which the magnetic field is $\vec{B}=B \hat{B}=\nabla \psi \times \nabla \theta$, and the flux line curvature vector is $\vec{k}=\hat{6} \cdot \nabla \hat{b}=9 \nabla \psi+10 \nabla \theta$. The covariant components $g$ and 6 will be called the normal and geodesic curvatures, respectively. Also, we have used a prime to denote differentiation along the field, $6 \cdot \nabla=\partial / 2 z$; $p_{1, \|}(B, \psi)=v(\psi) \hat{p}_{\perp, 11}(B)$ for the plasma pressure components perpendicular and parallel to $B ; x(\psi, \theta, 2), y(\psi, \theta, z)$ for flux lines; $i=\hat{G} \cdot \nabla \times \hat{b}$ for the parallel current per unit magnetic flux; $B_{v}(z)$ for the vacuum field strength; and $Q$ $\equiv \mathrm{B}^{2}+\mathrm{p}_{1}-\mathrm{p}_{11}$. The boundary conditions used to close the equations are $i=0$ at the ends of the system and $\vec{B}+\vec{B}_{v}$ laterally.

Equations (1) - (5) are still a very complicated set of coupled, nonlinear, third-arder partial differential equations for the unknowns $x$ and $y$. To solve, we further expand in powers of $B \equiv$ plasma energy density/vacuum field energy density. To $O(B)$ we find that the solution reduces to quadratures over known vacuum field quantities (functions of $z$ ) and over prescribed pressure profiles (functions of $\psi$ and $B$ ). We emphasize that the only ordering assumed is $\lambda^{2} \ll \beta<1$; the mirror ratio and flux surface ellipticity are arbjtrary. The constraint $\beta \gg \lambda^{2}$ is necessary because the vacuum fjeld drifts of particles trapped in the quadrupole end cells do not coincide with their drifts in the equilibrium field, with the result that isorrhopic [1] $\left[p_{1, \|_{2}}=p_{\perp, 11}(\psi, B)\right]$ pressure profiles may not exist for $B^{\prime}<\lambda^{2}$. 
As is clear from Eq. (4), it is a general feature of these equilibria that plasma currents flowing parallel to $\vec{B}$ are generated by pressure gradients in the presence of nonvanishing geodesic curvature. If these currents flow through the central cell they distart the flux surfaces that are circular at low $B$. If the currents are large or the central cell long, the distortions can be large enough to force flux surfaces to intersect material structures or, worse, to preclude the existence of equilibrium.

Fortunately, however, central-cell parallel currents can be greatly reduced or even eliminated, and the flux surface deformation concomitantly reduced, by proper iteration of magnet design with equilibrium calculations. He illustrate this in the case of a very long central cel1. To $O(B)$ the central-ce]l midplane flux-surface distortion assuming $v(\psi)=$ $1-\psi / \psi_{B}$, is

$$
\frac{\Delta r_{0}}{r_{0}}=k(\psi)+\frac{1}{3} \frac{\psi}{\psi_{B}}\left[\int_{0}^{l} \frac{d z}{B}\right] \frac{\left[\int_{l}^{L} \frac{d z}{B} \hat{p} g\right]^{2}}{\int_{l}^{L} \frac{\partial z}{B} \hat{p} h} \cos 4 \theta,
$$

Here $r_{0}$ is the midplane radius of the surface, $\psi=\Psi$ marks the plasma boundary, $\hat{\mathrm{p}}=\left\langle\hat{\mathrm{p}}_{1}+\hat{\mathrm{p}}_{1}\right) / 2$, and $\mathrm{g}(\mathrm{z})$ and $\mathrm{h}(\mathrm{z})$ are, respective]y, the odd and even parts of the vacuum curvature components: $\mathrm{A}_{v}=h(z)+g(z) \cos 2 \theta,{ }^{10} y=-2 \psi g(z) \sin 2 \theta$. The vacuum field is taken as axisymmetric for $|>|<\ell$ and nonax isymmetric for $\ell \leq|2| \leq L$, and terms of $v(1-\ell / L)$ have been neglected.

The equilibrium distortion of the vacuum surface as given by Eq. (6) consists of an axisymmetric component that arises from the well-digging effect of diamagnetic currents, and an octupole part arising from parallel currents. The axisymmetric component is proportional to $B$ in the central cell; the octupole component has contributions from both the transition region and the anchor, each scaling with the appropriate $\beta$, as well as a cross term which scales as $\sqrt{B_{C} B_{A}}$. To the same accuracy as Eq. (6), the parallel current throughout the axisymmetric region is

$$
i(0)=i(l)=2 \psi\left[\int_{\ell}^{L} \frac{d z}{B} \frac{\partial p}{\partial \psi} g\right] \sin 2 \theta
$$

Comparison of Eqs. (6) and (7) shows that on a given fux surface the octupole component is proportional to the square of the parallel current amplitude, which itself is proportional to the pressure-gradient-weighted flux tube average of the geodesic curvature over the non-axisymmetric region.

In an isolated quadrupole mirror celt, $g(z)$ is an odd function. When such a cell is connected to the solenoidal central cell of a tandem, however, this symmetry tends to be unbalanced by recircularizing coils in the transition region. Consequently, paraitel current flows from the end cell through 
the transition region (where it can be enhanced by additional locally unbalanced geodesic curvature) to the central cell, where it causes flux surface deformation. The key to reducing the distortions, therefore, lies in reducing the pressureweighted integral of $g$, that is, in optimaliy designing the structure of the vacuum geociesic curvature. In practice this is accomplished by alternating coil design changes with equilibrium caleulations until the central-cell parallel current is as small as possible, cons istent with other design constraints. An example of the result of this process is shown in Fig. 1. Plotted are the magnetic fieid strength $B$, the geodesic curvature 0 , and the parallel current $i$, for the MFTF- $B$ experiment. Also shown are several flux surface cross sections at the midplane of the central cell. The vacuum surfaces are shown dotted, while the equilibrium surfaces into which they are deformed are depicted with solid lines. (In [2] an algebraic error led to the opposite sign for the second term on the right side of Eq. (6), with the result that equilibrium flux surfaces had a "square" shape rather than the "diamonds" shown in Fig. 1d.)

\subsection{Finite-Orbit Corrections}

According to Eq. (6) the octupole component of the flux surface distortion scales with the length of the axisymmetric region. Therefore, if the central cell is very long, even a small parallel current can lead to apprec jable deformation [2-3]. For very large values of $\ell$, the analysis has to be modified by inclusion of finite-orbit effects, and of $\vec{E} \times \vec{B}$ drifts. Equation (4) is generalized to

$$
\begin{aligned}
B\left(\frac{Q}{B^{2}} i\right)^{\prime} & +\hookleftarrow \frac{\partial Q}{\partial \psi}=\gamma \frac{\partial}{\partial \theta}\left(\frac{\partial x}{\partial \psi} \frac{\partial^{2} x}{\partial \theta^{2}}+\frac{\partial y}{\partial \psi} \frac{\partial^{2} y}{\partial \theta^{2}}\right) \\
& =\frac{\partial}{\partial \psi}\left[\gamma\left(\frac{\partial x}{\partial \theta} \frac{\partial^{2} x}{\partial \theta^{2}}+\frac{\partial y}{\partial \theta} \frac{\partial^{2} y}{\partial \theta^{2}}\right)\right],
\end{aligned}
$$

where (for low B)

$$
\gamma=\frac{\partial \Phi}{\partial \psi}\left(-\rho \frac{\partial \Phi}{\partial \psi}+\frac{1}{2} B \frac{\partial M}{\partial \psi}\right) \text {. }
$$

$\Phi=$ ambipolar electrostatic potential, $\rho=$ density of mass, $M=$ density of gyrational angular momentum, summed over species. (The contribution to $M$ of any given species is $-2 m p / e B$, where $m=$ mass, $e=$ charge, $p_{1}=$ partial pressure, 1 component.) If $Y>0$, then the effect of the new term is to give. the flux surfaces an additional degree of rigidity. Let us assume $Y(\psi)=$ constant, for simplicity. Formula (6), implying linear growth of the octupole term as a function of 
$l$, is valid for $l \ll \ell_{c}$, where

$$
l_{c}=\frac{B_{0}^{2}}{Y} \int_{l}^{L} \frac{d z}{B} \hat{p h} \text {. }
$$

For $\ell \gg l$, on the other hand, the octupole distortion saturates at a finite level. If the simplest possible boundary condition is assumed (rigid wall at the lateral plasma boundary), then the resuit for $\ell \gg l_{c}$ is

$$
\frac{\Delta r_{0}}{r_{9}}=k+\frac{1}{30} \bar{\gamma}_{0}^{2}\left(\int_{l}^{L} \frac{d z}{B} \hat{\rho}\right)^{2} \frac{\psi}{\Psi_{B}}\left(1-\frac{\psi}{\Psi_{B}}\right) \cos 4 \theta .
$$

If $Y<0$, then a rotational instability sets in.

\subsection{Arbitrary $B$}

Equilibria computed by means of the $\beta$-expansion technique are reliable only so lang as the distortions of the vacuum flux surfaces are small. Furthermore, to quantify their accuracy it would be necessary to compute to next order in $B$, an apparentiy intractable problem. As an alternative, we have adapted to tandem-mirror geometry the reduced MHD technique used for calculating toroidal equilibria [4]. In this approach; the tensor-pressure MHD equations are expanded in powers of the paraxial parameter $\lambda$ to yield a set of coupled nonlinear equations of motion valid for arbitrary $B$. Equations for the flux coordinates $\psi$ and $\theta$ and a velocity stream function $U$ are solved numerically as an initial-value problem.

The paraxial expansion naturally separates the motion into three disparate timescales. On the fastest, compressionalAlfvêh-wave, timescale, the plasma is brought to perpendicular pressure balance, in which the equilibrium Equations (1) and (3) are satisiied. On the intermediate sound-wave timescale, parallel pressure balance, described by $\mathrm{Eq} .(2)$, is reached. Finally, on the slowest, shear-Alfvên-wave, timescale, the plasma responds to flux-line curvature, while maintaining the lower-order pressure-balance equilibrium. When equilibrium is attained on the slow timescale, Eqs. (4) and (5) are satisficd.

In our computations, we first obtain pressure balance perpendicular and parallel to $\vec{B}$. Perpendicular balance is found by solving the leading-order equations af motion, removing kinetic energy until equilibrium is reached.

Parallel balance is then obtained trivially from Eq. (2). Using the pressure-balance equilibrium as an initial condition, we then solve the higher-order shear-Alf vén-wave equations of motion [4]:

$$
\begin{aligned}
& \rho \frac{d}{d t}\left(\nabla_{1}^{2} U\right)=-B^{2} b \cdot \nabla\left(\frac{Q}{B^{2}} i\right)+\vec{k} \cdot \nabla Q \times \hat{b} \\
& \frac{\partial \psi}{\partial t}=\nabla U \times \nabla \psi \cdot \hat{b}_{v}, \quad \frac{\partial \theta}{\partial t}=\nabla U \times \nabla \theta \cdot \hat{b}_{v}
\end{aligned}
$$


where $U$ is a stream function in terms of which the flow velocity is $\vec{v}=$ DU $\times \vec{b}_{v}$, and $\vec{b}_{v}$ is a unit vector parallel to the vacuum field. Note that the vanishing of the right side of Eq. (12) at equilibrium is equivalent to Eq. (4).

We solve the equations numerically in finite-

difference form on a fixed rectangular grid. We assume appropriate symmetry, so that the grid needs to cover only one-eighth of the plasma volume. The lateral boundary conditions are those appropriate at a rjgid wall, viz., $P_{1}=P_{I I}=0$ and $U=0$, At the axia) boundaries we set $p_{1}=p_{\| I}=0$ and $\left(B_{V} U\right)=0$, the latter condition being equivalent to insulated ends. Kinetic energy is removed to pbtain a minimum-potential-energy state.

He have performed equilibrium calculations for two types of mirror configurations. In one, we considered a model quadrupole mirror similar to the end-cells of a tandem [4]. We foutd that if the plasma was interchange or ballooning unstable, exponentially growing nodes with a predominantiy $m=4$ structure rose nonlinearly to large amplitude [4]. When the plasma was MHD stable, on the other hand, we obtained wellconverged equilibria.

The model quadrupole vacuun field $c$ an be parametrized by $R$, the mirror ratio, and $\varepsilon=L[d(\ln x) / d z]_{z=0}(L=h a l f-l e n g t h$ of mirror, $x=$ symetry $p$ lane flux linel, which measures the ellipticity of the vacuum flux surfaces. Numerical stability results are shown in Fig. 2, which gives the critical value of $\varepsilon$ as a function of $B$ for two different mirror ratios. If $\varepsilon$ is less than the critical value, for a given $R$ and $B$, then the plasma is unstable. The $B=0$ i imits of the curves agree well with the low- $\beta$ interchange criterion. Finite $\beta$ is mildly destabilizing, but it is not difficult to find high-B stable iquilibria.

Tandem-mirror computations using the TMX $U$ vacuum field were performed in the same way. The $\hat{p}_{1}$ il(B) profiles were of the form given in [2], with $v(\psi)=\left(1-\psi_{3} \psi_{B}\right)^{2}$. In an equi-

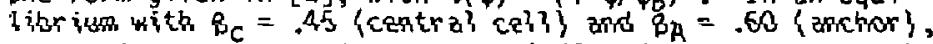
constant-pressure contours were simjlar in structure to those in Fig. 1d, with significant $m=4$ distortion. In the transition region, the ellipticity of vacuum flux surfaces was increased by an $m=2$ distortion.

To study the stability of tandems to low-m ballooning modes by this technique, it is necessary to relax the symmetry. The results of such computations will be given elsewhere.

\section{STABILITY}

Quadrupale-stabilized tandem mirrors rely for MHD stability an high pressure-weighting of the regions of favorable curvature (the quadrupole end cells) relative to those of unfavorable curvature. This guarantees stability to curvature-driven modes when the value of $B$ in the region of unfavorable curvature, $\beta_{c}$, is less than some critical value that depends on the value of $B$ in the end cells, $B_{A}$, and on the details of the magnetic geometry. To deteraine the 
critical value of $B_{C}$ it is necessary to formulate and solve the equation of motion for the modes of interest. At low $B$, the characteristic perturbations are electrostatic flute modes whose stability criterion is simple and well-known [5]. At higher values of $B$ magnetic-field perturbations make line bending possible; this allows modes to localize in regions of unfavorable curvature. It is the stability properties of such ballooning modes that ultimately determine the critical 8-values achievable in tandem mirrors.

\subsection{Moderate-m ballooning}

The analys is of ballooning modes is greatly simplified when the scale length for the variation of the perturbation perpendicular to $B$ is very much shorter than the scale length for variations along $\vec{B}$, as this assumption leads to a decoupling of the equation describing the parallel variation of the stream function $\phi$ from those descrjbing the perpendicular variation of $\phi$. Writing $\phi(\psi, \theta, z)=\phi(z) \exp \{S(\theta, \psi)$, one obtains a second-order ordinary differential equation to be solved for $\phi$ on each field line [6]. In the ideal MHD equations, the most unstable modes occur in the limit $|\nabla 5|+\infty$. In this limit, the perturbation is localized to a single fielc line, so it is only necessar:? to search for the first field line on which ballooning modes become unstable as $B_{c}$ is increased [7].

The introduction of inite-ion-Larmor-radius effects into the MHD equation [8] limits the values of $|\nabla S|$ for the most unstable perturbations. At finite values of $|\nabla S|$ the perturbation is no longer localized to a single field line. Hence, it is necessary to "sew" the solutions $\$(z)$ from neighboring field lines together in a self-consistent way to obtain both the mode structure in the $(\psi, \theta)$ plane and the global dispersion relation. We accomplish this by using the theory of semi-classical mechanics [9], essentially WKB theory generalized to many dimensions. The problem is then reduced to quantizing the two independent actions, $6 \mathrm{gs} \cdot \mathrm{dq}$, associated with the motion of a bal looning mode "ray" in the $(\psi, \theta)$ plane.

A numerical computation of the ray orbits and quantiza$t$ ion of the associated actions yields the stability boundary shown in Fig. 3 . Note that as the ion gyroradius, $a_{j}$, divided by the pressure-gradjent scale length, $R_{p}$, is increased, the critical value of $8_{c}$ increases substantially. The figure also shows that the value of $S_{\theta}$ (for axisymetric systems $S_{\theta}=m$ ) characteristic of the most unstable perturbation plumets to such small values, $S_{\theta} \leq 5$, that our large-m expansion fails well before we reach realistic values of $a_{j} / R_{p}\left(a_{j} / R_{p}=8 . \times 10^{-2}\right.$ for MFTF-B). This indicates that large-m bal looning modes will be suppressed by finite-1armor-radius effects in tandem-mirror experiments, and suggests that the most unstable modes will have very little structure in the $(\psi, \theta)$ plane. 


\subsection{Rigid Modes}

In the limit in which FLR effects completely dominate the energetics, only rigid displacements are possible. We analyze the stability of such modes by considering kise energy principle in the paraxial limit [1]. The displacement is convenientiy represented in terms of its Cartesian components as

$$
\vec{\xi}=u(z) \sigma \hat{x}+v(z) \tau \hat{y}
$$

where $\hat{x}$ and $\hat{y}$ are unit vectors, $\sqrt{B_{y}(0)} \sigma(z) \equiv x(\psi, \theta, z) /$ $x(\psi, \theta, 0), r B_{v}(0) r(z)=y(\psi, \theta, z) / y(\psi, \theta, 0)$, and $u$ and $v$ are arbitrary. The perturbed magnetic field inside the plasma corresponding to (14) is

$$
\delta \vec{B}_{\perp}=\frac{u^{\prime}}{\tau} \hat{x}+\frac{v^{\prime}}{\sigma} \hat{y} \text {. }
$$

In evaluating the energy integral, we use the geomatry of the vacuum field. We can then obtain the perturbed field in the vacuum region between the plasma and a conducting wall by solving Laplace's equation in confocal elliptic coordinates, requiring that the normal component of $\delta \vec{B}$ be continuous across the plasma boundary and vanish at the wall. the form

After integration over $\theta$ and $\psi$, the energy integral takes

$$
\begin{aligned}
\delta W=\pi \psi_{B} \int_{-L}^{L} d z\left[\frac { 2 } { B } \langle p \rangle \left(\sigma \sigma^{\prime \prime} u^{2}\right.\right. & \left.+\tau \tau^{\prime \prime} v^{2}\right)+\left(\frac{\langle Q\rangle}{B} \sigma^{2}+\eta\right) u^{\prime 2} \\
& \left.+\left(\frac{\langle Q\rangle}{B} \tau^{2}+\eta\right) v^{\prime 2}\right]
\end{aligned}
$$

where $\langle p\rangle \equiv \int_{0}^{\Psi_{B}} p d \psi / \psi_{B},\langle Q\rangle=\int_{0}^{\Psi_{B}} Q d \psi / \psi_{B}$,

$$
n=\frac{2\left(\psi_{W} / \psi_{B}\right)+\sqrt{4\left(\psi_{\mathrm{H}} / \psi_{B}\right)^{2}+B^{2}\left(\sigma^{2}-\tau^{2}\right)^{2}}+B(\sigma+\tau)^{2}}{2\left(\psi_{W} / \psi_{B}\right)+\sqrt{4\left(\psi_{W} / \psi_{B}\right)^{2}+B^{2}\left(\sigma^{2}-\tau^{2}\right)^{2}}-B(\sigma+\tau)^{2}},
$$

and $\psi_{w} . \Psi_{B}$ are the axial magnetic flux at the wall and plasma boundary, respectively. In Eq. (16), the term proportional to $\langle p\rangle$ comes from the curvature drive, while the others represf $a t$ the perturbed magnetic energy, with the individual terms multiplied by $\langle Q\rangle$ arising inside the plasma and those multiplied by $n$ coming from the vacuum region.

We obtain Euler-Lagrange equations by varying ow with respect to $u$ and $v$ independentiy. The result is

$$
\frac{d}{d z}\left[\left(\frac{Q\rangle}{B} \sigma^{2}+\eta\right) \frac{d u}{d z}\right]-\frac{2}{8}\langle p\rangle \sigma \sigma^{\prime \prime} u=0 \text {, }
$$


and $v(z)=u(-z)$, by symmetry. Eq. (17) is identical to the "infinite-m" ballooning-mode equation at marginal stability, except for the presence of the $\pi$-term and the substituion of $\langle Q\rangle$ and $\langle p\rangle$ for $Q$ and $p$. Since $1 \leq n \leq \infty$, therefore, rigid modes are inherently more stable than "infinite-m" modes. As the wall approaches the plasma $(n \rightarrow \infty)$ the mode becomes flute-like, and the interchange limit is recovered. An illustration of this behavior in the case of TMX-Upgrade is given in Fig. 4, Shown are marginal-stability boundaries for two different wal] positions, and the dependence of the critical $\left\langle\beta_{C}\right\rangle$ on wall position for several values of $\left\langle B_{A}\right\rangle$.

\section{REFERENCES}

[]] NEWCOMB, W.A., J. Plasma Physics 26 (1981) part 3, 529.

[2] PEARLSTEIN, L.D., KAISER, T.B., and NEWCOMB, W.A., Phys. Fluids 24 (1981) 1326.

[3] STUPAKOV, G.V., Fiz. Plasmy 5 (1979) 87) [Sov. J. Plasma Phys. $5(1979) 486]$.

[4] STRAUSS, H.R., to appear in Nuclear Fusion (1982).

[5] HALL, L.S. and MCNAMARA, B., Phys. FTuids 18 (1975) 552.

[6] CONNOR, J.W., HASTIE, R.J., and TAYLOR, J.B., Phys. Rev. Lett. 40 (1978) 396; GLASSER, A.A., (Proc. The Finite Beta Theory Workshop, Varenna, 1977) NTIS, Springfield (1979) 55.

[7] KAISER, T.B, and PEARLSTEIN, L.D., Lawrence Livermore Nationa] Laboratory Report UCRL-87659 (1981) [submitted to ihys. Flujids].

[8] TANG, H.M. and CATTO, P.J., Phys. Fluids 24 (1981) 1374;

LEE, X.S, and CATTO, P.J., Phys. Fiuids $24(1981) 2010$.

[9] PERCIVAL, 1.C., Adv. Chem. Phys. 36 (1977) 1. 


$$
-4 i-
$$

\section{FIGURE CAPTIONS}

Fig. 1. Several functions characterizing equilibrium in MFTF-B, a) Vacuum magnetic field strength as a function of distance along magnetic axis, $z$. The central cell, centered at $z=0$, is flanked by the axicell, transition region and MHD anchor. b) Geodesic curvature on a flux 'ine $\psi=\psi_{B}, \theta=\pi / 4$. Vertical dotted Tines mark extrema of $B_{v}(z)$. c) Parallel current on a flux line with $\theta=\pi / 4$. Note that current leaving anchor is effectively zero, while that leaving transition region is nearly so. d) Fluxa surface cross sections at $z=0$. Equilibrium surfaces (solid Tines) show octupole distortion, while vacuum surfaces (dotted lines) are circular.

Fig. 2. Ellipticity parameter $\varepsilon$, in model quadrupole-mirror cer? as a function of marginaly stable a for two different mirror ratios. The equilibrium is unstable to ballooning-interchange modes below the curves.

Fig. 3. Stability boundaries and mean azimuthal mode number, $\left\langle S_{\theta}\right\rangle$, for an axicell MFTF-B design. The value of $B$ in the MHD anchor cell is held fixed at $\beta_{A}=0.55$. Note the substantial increase in $\beta_{c}$ with $a_{j} / R_{p}$ unti? the calculation begins to break down at $\left.a_{j} / R_{p} \simeq 3 \times 1\right)^{-3}$. Tandem mirror experiments typically have $a_{j} / k_{p}=0.1$.

Fig. 4. Marginal stability boundaries for $r$ gid modes in TMXUpgrade. a) $\left\langle\beta_{A}\right\rangle$ as a function of $\left\langle\beta_{C}\right\rangle$ for two different wall positions, with the low- $\beta$ flute limit shown for comparison. The equilibrium is stable to the left of the curves. b) $\left\langle\beta_{C}\right\rangle$ as a function of wall position for three values of $\left\langle\beta_{A}\right\rangle$. The equilibrium is stable below the curves. 

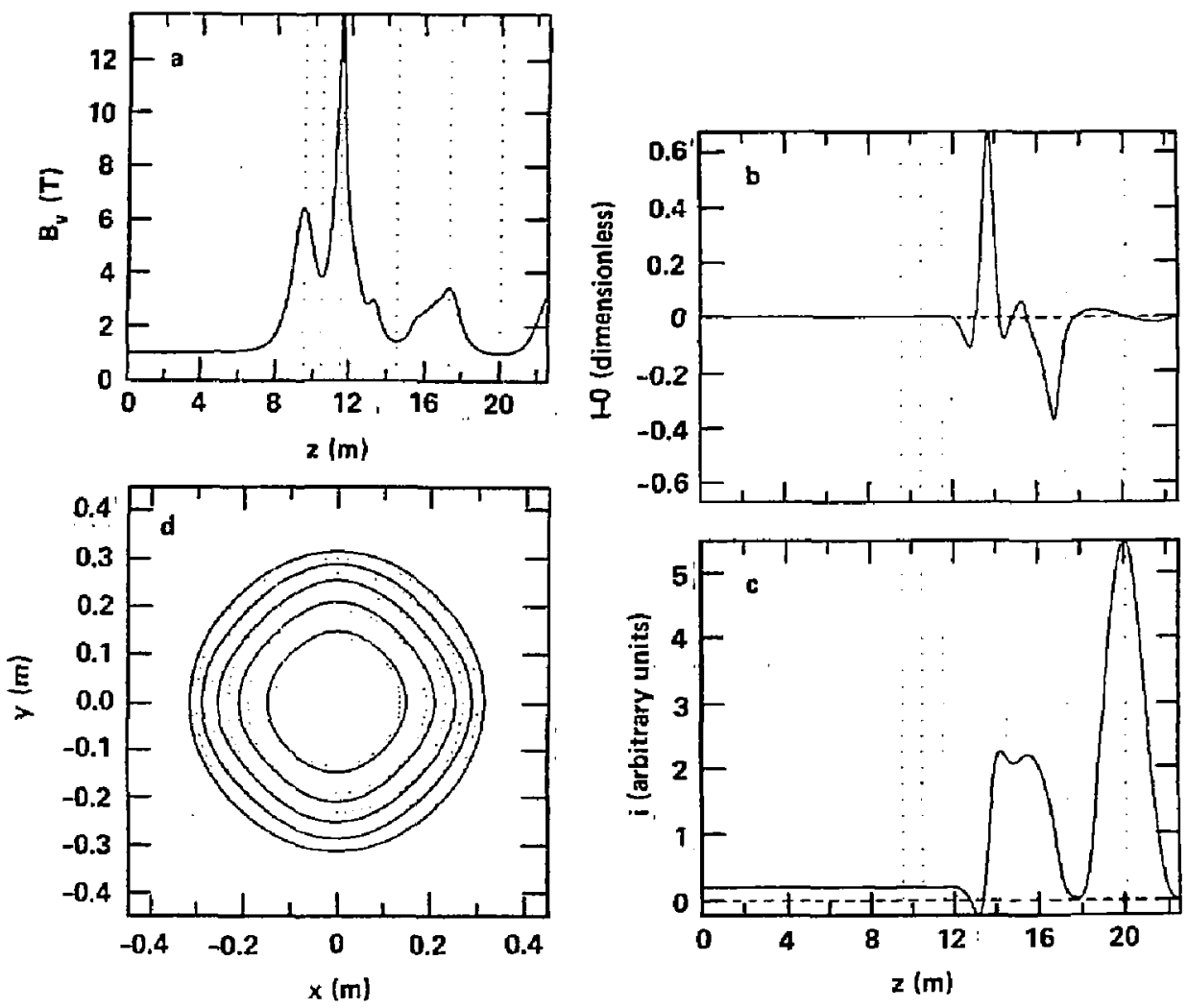

Fig. 1. Several functions characterizing equilibrium in MFTF-B. a) Vacuum magnetic field strength as a function of distance along magnetic axis, $z$. The central cell, centered at $z=0$, is flanked by the axicell, transition region and $M H D$ anchor. b) Geodesic curvature on a flux line $\psi=\psi_{B}, \theta=\pi / 4$. Vertical dotted lines mark extrema of $B_{v}(z)$. c) Parallel current on a flux line with $\theta=\pi / 4$. Note that current leaving anchor is effectively zero, while that leaving transition region is nearly so. d) Fluxsurface cross sections at $z=0$. Equilibrium surfaces (solid lines) show octupole distartion, while vacuum surfaces (datted lines) are circular. 


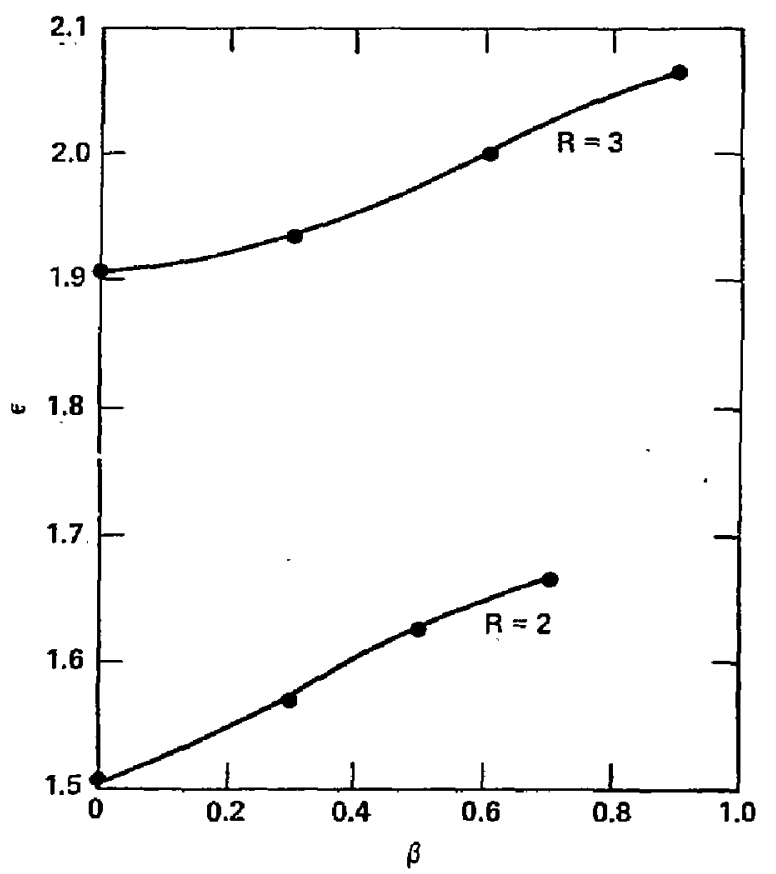

Fig. 2. Ellipticity parameter $\epsilon$, in model quadrupole-mirror cell as a function of marginally stable $B$ for two different mirror ratios. The equilibrium is unstable :o ballooning-interchange modes below the curves. 


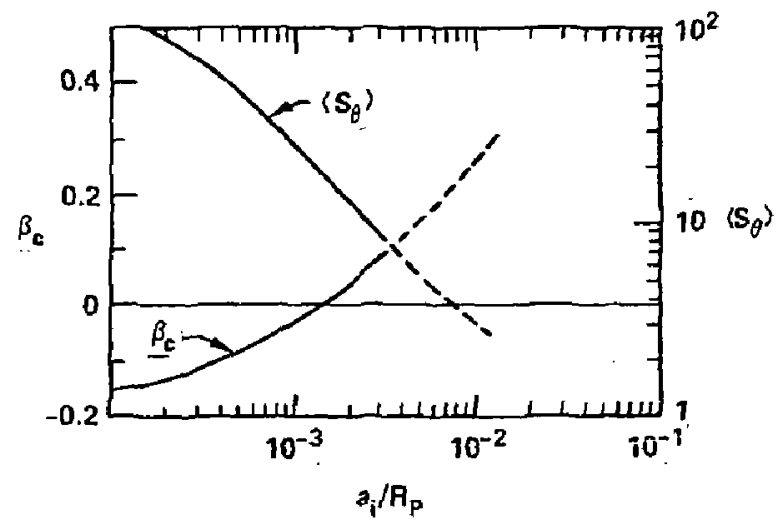

Fig. 3. Stability boundaries and mean azimuthal mode number, $\left\langle\mathrm{S}_{\theta}\right\rangle$, for an axicell MFTF-B design. The value of $B$ in the MHD anchor cell is held fixed at $B A=0.55$. Note the substantial increase in $\beta_{C}$ with $a_{j} / R_{P}$ until the calculation begins to break down at aj $R_{p}=3 \times 10^{-3}$. Tandem mirror experiments typically have $a_{j} / k_{p}=0.1$. 

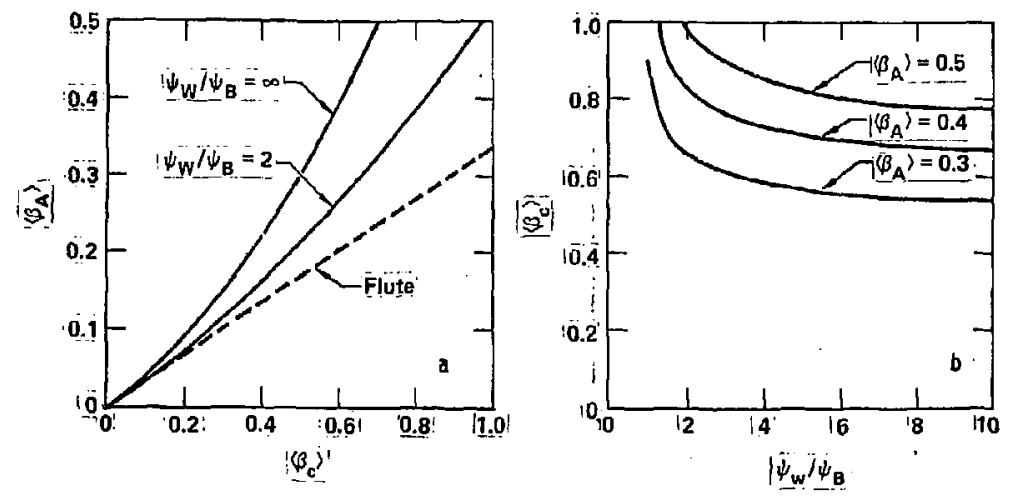

Fig. 4. Marginal stability boundartes for rigid modes in TMXUpgrade. a) $\left\langle\beta_{A}\right\rangle$ as a function of $\left\langle\beta_{C}\right\rangle$ for two dffferent wall posftions, with the low- $\theta$ flute limit shown for comparison. The equilibrium is stable to the left of the curves. b) $\left\langle\beta_{C}\right\rangle$ as a function of wall position for three values of $\left\langle B_{A}\right\rangle$. The equilibrtum is stable below the curves. 
$-46-$

APPENDIX C: A Comparison of Length-Sensitive Physics Parameters in MFTF-B and Reactor-Scale Devices 
APPENDIX C: A Comparison of Length-Sensitive Physics Parameters in MFTF-B and Reactor-Scale Devices

MFTF- $B$ is intended as the final physics step before a major DT facility that is to be aimed principally at engineering and advanced fusion-technology development. The parameters of the MARS study ${ }^{C l}$ serve to illustrate such a device. MFTF-B has then to validate the physics models and scaling laws that have been used in MARS-type reactor assessments. A number of physics questions need to be answered in this connection. These questions deal with: whether the scaling laws are right for the physics issues that have been identified and are considered to be in hand, whether uncertain or potentially troublesome issues on the reactor scale can be explored in MFTF-B, and whether there might be physics issues that have been missed.

MFTF-B was first conceived and designed as a facility having an orderone-second axial canfinement time, the total confinement time required for a reactor. Achieving it in MFTF-B will allow assessment of such issues as transport, impurity accumulation, hot electron distribution control, and near steady-state plasma operation and technology. Axial confjnement depends on the plug properties and the central cell temperature. Most of the new physics to be explored is associated with performance of the plugs and their improvement, e.g., by thermal barriers. The approach has been to develop near-reactor grade plugs in MFTF-B, which has a relatively shart central cell. Larger, reactorgrade machines would then require only lengthening the central cell, provided the MFTF-B central cell is adequate to give the necessary scaling information.

To the extent that the aspect ratios and dimensionless physics ratios for the smaller MFTF-B central cell can be made nearly equal to those of the reactor, the scale-up can be made with some confidence. Significant 
differences in these ratios would raise the possibility that the MFTF-B central cell might be operating in a different physics regime, either more or les 5 favorable, for example, with respect to its $\beta$-holding capability or its stability to a particular mode.

The marked difference between MFTF-B and a MARS-type device is the length of the central cell, both absolutely and in ratio to the radius. This was true in the originaT A-cell configuration, and it is even more dramatic in the axicelt configuration described in the text. The axicell design is much closer in concept to the MARS reactor design than was the A-cell design; this was the chief motivation for the change. The reduction in solenoid length from the A-cell design to the axicell design came about because of a ground rule that the modification should not increase the project cost or schedule. In practice, this meant not changing the location of the yin-yang magnet sets. By adding the axicell coils between the yin-yangs and the central cell, when allowance has been made for the transitions between cells, the solenoid length has been reduced from $26 \mathrm{~m}$ to $13.75 \mathrm{~m}$, while the mirror-to-mirror length of the axisymmetry region of the central cell plus axicells is $20.3 \mathrm{~m}$. For a MARS reactor, the solenoid length is $150 \mathrm{~m}$. This disparity quite properly raises the question as to the adequacy of the central cell to provide the necessary scaling information. Other dimensions for the various machines, such as radius and plug/anchor dimensions, differ by less than a factor of two.

A number of important parameters depend on some measure of the machine Jength, although their detailed weightings of line averages differ. For many of the important physics considerations, it is the total axisymetric region enters as the most representative length, $i . e .$, the $20.3 \mathrm{~m}$ figure cited above. These physics issues include, for example, the ion azimuthal drift-per-bounce as it determines transport, the influence of parallel current as it affects equilibria, and the effect of rotation on low frequency stability. 
The only parameter that depends directly on the effective length (or volumel of the central cell is the calculated equivalent fusjon power produced in an effective-Q calculation. (The effective length is that of an equalvolume cylinder having the same midplane radius.) While $Q$ is of significance itself as a performance milestone, as noted above the more signifjcant physics measure of confinement is the confinement time. Larger $Q$ would come with longer central cell.

As the design has proceeded, the central cell length has decreased in two steps. In April, the $6 \mathrm{~m}$ transition between the yin-yang anchor and axice 1 was unsatisfactory on issues of transport and equiliorium currents. In that design, the effective central cel1 length was $76.5 \mathrm{~m}$ and full length of the axisymmetric region was $23 \mathrm{~m}$. The change was accepted by the review panel in part because the one-second confinement time had been preserved and the A-cell design confinement parameter $Q$ had been maintained due to an increased central cell density offsetting the shortened central cell.

As described in the text the final transition design in August, which satisfied the transport and equilibrium issues, required $7.25 \mathrm{~m}$ transition regions at each end. At the same time the axicell coils were increased somewhat in radius for engineering reasons, giving more taper to the central cell, a final central cell effective length of $12.6 \mathrm{~m}$, and an axisymmetric region of $20.3 \mathrm{~m}$.

The August transition coil design improved certain important physics parameters compared to the April design. A comparison of the parameters . ur the three cases is shown in Table Al. Note that the principal improvement occurs in the transverse lifetime. Only in the August design does this lifetime exceed the axial lifetime (assuming reduction of the radial electric field in the transition regions as discussed in Appendix $A$ ), thereby reaping one of the advantages of the axicell configuration. 
Table A.. Comparison of Parameters

\begin{tabular}{|c|c|c|c|}
\hline Center cell & A-cel1 & $\begin{array}{c}\text { April } \\
\text { axicel }\end{array}$ & $\begin{array}{l}\text { August } \\
\text { axicel1 }\end{array}$ \\
\hline Number of solenoid coils & 14 & 14 & 12 \\
\hline Physical solenoid length, $L_{c}^{\star}(m)$ & 26 & 16.25 & 13.76 \\
\hline Effective solenoid length, $L_{c}(m)$ (a) & 32 & 16.5 & 12.6 \\
\hline Axisymmetric length, $L_{\text {sgm }}(m)^{(b)}$ & 230 & 23 & 20.3 \\
\hline Plasma radius, $r_{p}(m)$ & 0.56 & 0.30 & 0.30 \\
\hline Plasma volume, $L_{c} \pi r_{p}^{2}\left(m^{3}\right)$ & 31.5 & 4.27 & 3.56 \\
\hline Number of jons $\left(10^{20}\right)$ & 6.3 & 2.0 & 1.7 \\
\hline$r_{p} / \rho_{i}$ & 24 & 13 & 13 \\
\hline Column aspect ratio $L_{c} / r_{p}$ & 57 & 50 & 42 \\
\hline$T_{\text {ic }}\left(\mathrm{keV}^{\mathrm{V}}\right)$ & 15 & 15 & 15 \\
\hline$T_{e c}(k e v)$ & 9 & 9 & 9 \\
\hline$n_{c}^{e c}\left(10^{73} \mathrm{~cm}^{-3}\right)$ & 2 & 4.8 & 4.8 \\
\hline$B_{C}(T)$ & $\mathrm{T} .0$ & $1.0^{(c)}$ & $1.0^{(c)}$ \\
\hline Coilisionality $L_{C} / m f p$ & 0.0004 & 0.0004 & 0.0003 \\
\hline Transverse lifetime, $\tau_{t}(s)^{(d)}$ & 0.2 & 0.01 & 1.9 \\
\hline Axial Tifetime, $\tau_{11}(s)^{(d)}$ & 2.5 & 1.0 & 1.0 \\
\hline $\begin{array}{l}\text { Transport regime } \\
n \tau_{1}\left(\mathrm{~cm}^{-3} s\right)^{(d)} \\
n \tau_{\|}\left(c,-{ }^{3} s\right)(d)\end{array}$ & $\begin{array}{l}\text { banana } \\
4 \times 10^{12} \\
5 \times 10^{13}\end{array}$ & $\begin{array}{l}\text { stochastic } \\
5 \times 10^{11} \\
5 \times 10^{13}\end{array}$ & $\begin{array}{l}\text { banana } \\
10^{14} \\
5 \times 10^{13}\end{array}$ \\
\hline$\langle\beta\rangle_{\max }$ - fluting $(e)$ & 0.20 & 0.49 & 0.26 \\
\hline$\langle\beta\rangle_{\max }-$ ballooning $(e)$ & 0.15 & 0.31 & 0.18 \\
\hline$Q_{c}(f)$ & 0.6 & 0.6 & 0.6 \\
\hline
\end{tabular}


Table Al. (continued)

(a) The effective solenoid length, $L_{c}$, is the length of an equivalent uniform plasma cylinder. It may be smaller or larger than the solenoid length. The main significance of $L_{c}$ is in calculating $Q$; other physics parameters depend on length in more complicated ::ays.

(b) Several physics issues depend on the distance between non-symmetric regions. Here as a representative, such length $L_{\text {sym }}$ is defined as the distance between the outer mirror peaks of the axicells, and similarly for the A-cell.

(c) Because of the closer solenoid coil spacing in the axicell cases, $B_{C}$ can be increased to $1.6 \mathrm{~T}$ if desired.

(d) The $\tau$ and $n x$ values are for particle lifetimes. For the August axicell design (with $n \tau_{1}>n \tau_{11}$ ) the corresponding center cell energy confinement parameter is $n \tau_{E}=1.7 \times 10^{13} \mathrm{~cm}^{-3} \mathrm{~s}$.

(e) Volume average beta limits.

$(f)_{Q_{c}}(=$ center cell fusion power/heat leakage) is a measure of heat confinement in the center cell; if alphas are well confined, $Q_{C}=5$ is ignition. 
These parameters give rise to several scaling ratios that can be used to compare the physics performance of different size machines. These ratios are discussed below, and their numerical values for the A-cell and axicell designs of MFTF-B and the MARS study are given in Table $A 2$.

Azimuthal drift per bounce $\Delta \theta: \Delta \theta$ is the azimuthal precession of an inn while passing through the axisymmetric region. It is driven by the radial electric field; to avoid stochastic transport, $\Delta \theta$ should be kept $\leqslant 2 \pi$. Define the ExB frequency by int:oducing a parameter $\alpha_{E}$,

$$
\Omega_{E}=\alpha_{E} \frac{2 c T_{e}}{e B r_{c}^{2}}
$$

Then $\alpha_{E}=\Phi_{C} / T_{e}$ corresponds to a parabolic profile having ground potential at the surface $r_{c}$. This is a likely upper timit for $\alpha_{E}$; lower values imply some measure of poteriial profile control. Then

$$
\Delta \theta=\alpha_{E} \sqrt{\frac{T_{e}}{T_{i}}} \frac{\rho_{s} L_{s y m}}{r_{c}^{2}}
$$

where $\rho_{s}^{z}=2 T_{e} / m_{i} \Omega_{i}^{2}$, and $L_{s y m}$ is the effective length of the axisymmetr ic region.

Rotational sensitivicy: A full theory of stability to rotation modes has not been developed for non-axisymmetric geometry; therefore, we can only estimate and parameterize tho stabilizing effect of the anchors. For a mode to isolate away from the ancior region requires $k_{\| 1}^{2} v_{A}^{2} \geq \frac{1}{4} \delta_{E}^{2}$ with $k_{\|}=\pi / L_{s} y m^{\text {, }}$ giving

$$
\beta_{\mathrm{e}}<\frac{4 \pi^{2}}{a_{E}^{2}}\left(\frac{r_{c}^{2}}{\rho_{s} L_{s, y m}}\right)^{2}
$$


where $\beta_{\mathrm{e}}$ is the central cell electron cantribution to $\beta$. If this condition were to be violated, the central cell woutd be unstable irrespective of the anchors. To compare rotation versus anchoring in different machines, we use the ratio

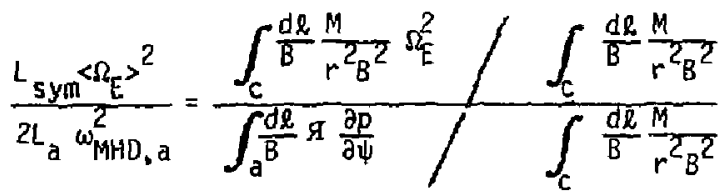

$$
\begin{aligned}
& \approx \alpha_{E}^{2} \frac{{ }^{B_{e}}}{B_{a}} \frac{B_{c}}{B_{a}} \frac{\rho_{s}^{2}}{r_{c}^{2}} \frac{R_{c_{1} a}}{r_{a}} \frac{L_{s y m}}{L_{a}}
\end{aligned}
$$

where $R_{C, a}$ is the radius of curvature in the anchor, and $M$ is the mass density. Smallness of this parameter is not directly a stability criterion, but it can be used to determine whether two machines will be in the same regime vis-a-vis rotation.

Irapped particle modes: According to recent theory the significant parameter determining stability to trapped particle modes in the HARS-type design is measured by the fraction of ians that communicate betwean regions of good and bad curvature.

$$
A=\frac{2\left(L_{t}+L_{a} j n_{t}\right.}{L_{s y m} n^{n}}\left(\frac{r_{c}}{\rho_{i}}\right)^{2} \gg 1,
$$

with A preferably greater than 5 or so, $n_{t}$ is the density in the transition region.

m=1 curvature-driven MHO ballooning modes: The effective length is automatically included in our balloon cade which calculates stability limits using aciual magnet fields. As regards scaling to longer central cells, we have 
compared calculations of two magriet sets, having identicle end cells, but one a MFTF-scale and the other a MARS-scale central cell. There was little difference in the calculated $B$ limits. This will be typical of double-ellipse transition designs, for nulling the average of to also nulls the azimuthally dependent part of $\boldsymbol{F}$. Then, at a given $\theta$, the two ends look nearly the same, and a perturbation cannot become mare unstable for bending in the central cell.

Equilibria: Acceptable values of non-vanishing parallel current in the central cell in MFTF-B would become quite unacceptable in a long central cell, for the distortion scales as currant times length. However, in the transition magnet design we were able to null the parallel current to arbitrary accuracy, so this consideration should only give a constraint on the precision of current nutling.

TABLE A2

Length Dependent Physics Parameters

\begin{tabular}{|c|c|c|c|}
\hline PARAMETERS & $A-C E L L$ & AXICELL & MARS \\
\hline Aspect ratio $\left(\mathrm{L}_{\mathrm{sym}} / \mathrm{r}_{c}\right)$ & 57 & 60 & 350 \\
\hline$r_{c} / o_{s}$ & 31 & 7 & 52 \\
\hline$L_{s y m} \rho_{s} / r_{c}^{2}$ & 1.8 & 3.5 & 7 \\
\hline$\Delta \theta$ per bounce & $1.4 \alpha_{E}$ & $2.7 \alpha_{E}$ & $5.9 \alpha_{\mathrm{E}}$ \\
\hline$B_{e}$ rotation limit & $12 / \alpha_{E}^{2}$ & $3.2 / \alpha_{E}^{2}$ & $.8 / \alpha_{E}^{2}$ \\
\hline$L_{s y m}\left\langle S_{E}^{2}>/ 2 L_{a} \omega_{M H D, a}^{2}\right.$ & $.003 \alpha_{E}^{2}$ & $.05 \alpha_{E}^{2}$ & $.04 \alpha_{E}^{2}$ \\
\hline$A^{*}$ (trapped particle modes) & 25 & 19 & 26 \\
\hline
\end{tabular}

$\star A$ is calculated for $n_{t} / r_{c}=0.1$ in all cases. Determination of the A-value required is important. 
$-55-$

Values of $a_{E}<6$ would represent some degree of potential profile flattening or control. In a reactor $1<\alpha_{E}<2$ would be required, based on the transport. $\Delta \theta$ and limiting $\beta_{e^{*}}$ MFTF-B is close enough to explore the feasibility of this.

\section{Summary of Physics Sensitivity to Length}

For all physics considerations except the effective Q calculation, the appropriate physical length of the central cell. is that of the axisymmetric region, although the proper averages can vary for different parameters. In the MFTF-B axicell design, this physical length of about $20 \mathrm{~m}$ was set by the required $7.25 \mathrm{~m}$ transition regions at each end. All identified physics parameters are within a factor of two between MFTF-B and MARS for the norma 7 operating scenerios. Some variation in the MFTF-B parameters could also be obtained by departing from the base MARS-mode scenerio. 


\section{REFERENCES}

1. The recommendations of this Review Pane 1 were contained in a Memorandum to W. R. Ellis, Office of Fusion Energy, Department of Energy, April 30, 1982.

2. L. D. Pearlstein, T. B. Kaiser, and W. A. Newcomb, Phys. Fluids 24,1326 (1981).

3. This condition was originally recognized by $D$. E. Baldwin and $L .0$. Pearlstein (unpublished) and recently by J. R. Myra, D. A. D'Ippolito, and P. J. Catto, SAI-254-82-174-LJ/PRI-44 (1982).

4. L. D. Pearlstein, T. B. Kaiser, and W. M. Nevins, manuscript in preparation; see also R. H. Bulmer, et al., IAEA Preprint in Appendix $B$.

C1. MARS Interim Design Report, B. G. Logan, C. Henning, et al., in preparation. 


\section{DISCLAIMER}

This documeat was prepared as an account of work sponsored by an apency of the United Sthies Government. Neither the United States Government nur the Unirendity of Cullfomin nor any uf their employees, mahes

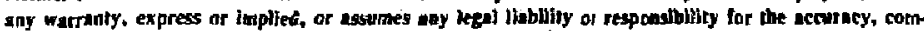
pleteness, of usefulness of any information, nppurntus, product, or procesy disclosed, or tepresents that lis use would not infringe privately owned rights. Reference herein to any specific commerclal products, process, ar service by trade name, Iradenark, mamifacturet, or otherwise, dots not necessintly consticuice or imply his endorsement, recommendation, or favoring by the United States Government of the University of Callformte. The viens and opinions of authors expiessed herein do wot necessarily state of reffort those of the United States Covernment therenf, and shall not be used for adrertising or product endorsement purposes.

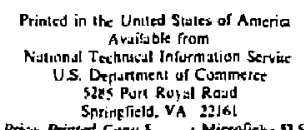

\begin{tabular}{lrrrr} 
Page Range & $\begin{array}{c}\text { Domestic } \\
\text { Price }\end{array}$ & Page Range & $\begin{array}{r}\text { Domestic } \\
\text { Price }\end{array}$ \\
\cline { 5 - 5 } $001-025$ & $\$ 5.00$ & & $326-350$ & $\$ 18.00$ \\
$026-050$ & 6.00 & $351-375$ & 19.00 \\
$051-075$ & 7.00 & $376-400$ & 20.00 \\
$076-100$ & 8.00 & $401-425$ & 21.00 \\
$101-125$ & 9.00 & $426-450$ & 22.00 \\
$126-150$ & 10.00 & $451-475$ & 23.00 \\
$151-175$ & 11.00 & $476-500$ & 24.00 \\
$176-200$ & 12.00 & $501-525$ & 25.00 \\
$201-225$ & 13.00 & $526-550$ & 26.00 \\
$226-250$ & 14.00 & $551-525$ & 27.00 \\
$251-275$ & 15.00 & $526-550$ & 28.00 \\
$276-300$ & 16.00 & $601-11 p 1$ & \\
$301-325$ & 17.00 & &
\end{tabular}

1 Add 2.00 for each additional 25 page increment from 601 pages up. 
Technical Information Department - Lawrence Livermore Laboratory University of California - Livermore, California 94550 